\title{
The generation of Tollmien-Schlichting waves by free-stream turbulence in transonic flow
}

\author{
A. I. Ruban $[$ ! H. Broadley
}

Received: 2 October 2020 / Accepted: 24 April 2021 / Published online: 17 June 2021

(C) The Author(s) 2021

\begin{abstract}
This paper studies the generation of Tollmien-Schlichting waves by free-stream turbulence in transonic flow over a half-infinite flat plate with a roughness element using an asymptotic approach. It is assumed that the Reynolds number (denoted $\mathrm{Re}$ ) is large, and that the free-stream turbulence is uniform so it can be modelled as vorticity waves. Close to the plate, a Blasius boundary layer forms at a thickness of $O\left(\operatorname{Re}^{-1 / 2}\right)$, and a vorticity deformation layer is also present with thickness $O\left(\mathrm{Re}^{-1 / 4}\right)$. The report shows that there is no mechanism by which the vorticity waves can penetrate from the vorticity deformation layer into the classical boundary layer; therefore, a transitional layer is introduced between them in order to prevent a discontinuity in vorticity. The flow in the interaction region in the vicinity of the roughness element is then analysed using the triple-deck model for transonic flow. A novel asymptotic expansion is used to analyse the upper deck, which enables a viscous-inviscid interaction problem to be derived. In order to make analytical progress, the height of the roughness element is assumed to be small, and from this, we find an explicit formula for the receptivity coefficient of the Tollmien-Schlichting wave far downstream of the roughness.
\end{abstract}

Keywords Boundary layer receptivity $\cdot$ Free-stream turbulence $\cdot$ Transonic flow

\section{Introduction}

Laminar-turbulent transition is a phenomenon which has been studied extensively by researchers for over a century, beginning with the pioneering experiments of Reynolds in the late nineteenth century. It is an extremely complicated process, which begins when small perturbations from the free-stream flow penetrate into the boundary layer and interact with roughness on a body surface, producing Tollmien-Schlichting waves. Tollmien-Schlichting waves are instability modes that travel downstream of a roughness element; however, they must often travel far downstream of this roughness before they cause transition to turbulent flow. In quiet flows (such as those seen in aerodynamic applications), the amplitude of these waves is at first too small to cause transition from laminar to turbulent flow. However, when the Tollmien-Schlichting waves grow in amplitude in the boundary layer, they eventually produce

A. I. Ruban $(\bowtie)$

Department of Mathematics, Imperial College London, 180 Queen's Gate, London SW7 2BZ, UK

e-mail: a.ruban@imperial.ac.uk

H. Broadley

School of Mathematics, University of Manchester, Manchester M13 9PL, UK 
nonlinear effects which are quickly followed by a transition to turbulent flow. The study of laminar-turbulent transition met a major obstacle when it was found that the transition would not necessarily take place at the same point in two experiments which used the same principal similarity parameters. For example, a model of an aircraft wing tested in different wind tunnels, with the same Mach number and Reynolds number of flow, would have different points at which the flow changed from laminar to turbulent.

Receptivity theory was introduced in order to account for this discrepancy, which is mostly due to the difference in perturbations present in the oncoming flow. Its aims are to describe the generation and propagation of TollmienSchlichting waves in the boundary layer, as well as to predict the point at which the amplitude becomes large enough to cause laminar-turbulent transition. In order to do this, it is necessary to identify the external perturbations which penetrate into the boundary layer and the external perturbations that do not. The objective of experimental works such as those performed by [1] was to study different types of perturbations and find which respective category each fell into. Examples of external perturbations that do penetrate into the boundary layer include acoustic waves; free-stream turbulence; and entropy waves. In this paper, Tollmien-Schlichting waves generated by a combination of free-stream turbulence and wall roughness are studied.

The effect of external acoustic noise on Tollmien-Schlichting wave generation in the boundary layer has been extensively studied for the past 40 years, beginning with [2,3]. However, neither of these papers predicted that Tollmien-Schlichting waves could be effectively produced by acoustic waves. Their work was extended independently by [4,5], who demonstrated that the strict resonance conditions, which have to be satisfied in order for perturbations to penetrate into the boundary layer and, therefore, generate Tollmien-Schlichting waves, of [6] can be applied to receptivity theory. In simple mechanical systems, resonance conditions are often easy to satisfy; for example, for a simple spring system, the only resonance condition is that the frequency of external forcing is the same as the natural frequency. For Tollmien-Schlichting waves, however, we require both the frequency and the wave number of external perturbations to match those of the natural oscillations in the boundary layer. References $[4,5]$ considered incompressible flow, and found that in order for both resonance conditions to be satisfied in the presence of acoustic waves, the frequency of the waves had to be of $\mathrm{O}\left(\mathrm{Re}^{-1 / 4}\right)$ and that wall roughness had to be introduced; otherwise, the wavelength of the perturbation was too large. Under these conditions, the amplitude of Tollmien-Schlichting waves was calculated. More recently, [7] studied the generation of Tollmien-Schlichting waves by acoustic noise in compressible transonic flows, using the triple-deck model of $[8,9]$.

The effect of free-stream turbulence on instabilities arising in the boundary layer has also been the subject of investigation. Reference [10] considered how uniform free-stream turbulence, taking the form of vorticity waves, could penetrate into the boundary layer in incompressible flow. The vorticity waves that form the uniform turbulence can also be thought of as convective gusts. These do not automatically satisfy the impermeability condition, which led Hunt and Graham to introduce a new 'vorticity deformation layer' of thickness $\mathrm{O}\left(\mathrm{Re}^{-1 / 4}\right)$. Further work by [11] analysed the effect of free-stream turbulence on the boundary layer, and concluded that vorticity waves do not penetrate far enough into the boundary layer to generate Tollmien-Schlichting waves. However, [12] demonstrated that this is not the case, despite the fact that pressure perturbations do not penetrate into the boundary layer. The authors introduced vorticity waves to incompressible flow over a half-infinite flat plate with roughness and solved the resulting viscous-inviscid interaction problem to find the amplitude of Tollmien-Schlichting waves produced.

The scope of the present study is to extend the analysis of [12] to transonic flow. Free-stream turbulence will be assumed to be uniform and, therefore, modelled by sinusoidal vorticity waves. Solutions to the Navier-Stokes equations will first be sought upstream of the roughness element where the main regions to be studied are the unperturbed flow region, the vorticity deformation region, and the classical boundary layer. Due to the lack of pressure perturbations outside of the boundary layer, it is necessary to introduce a transition layer in which vorticity waves decay in order to prevent a discontinuity in vorticity. Following this, the flow in the interaction region forming in the vicinity of the roughness will be studied. To accurately model the flow in the neighbourhood of the roughness, the triple-deck model with scalings given by $[8,9]$ will be used. The main difficulty is in the upper tier of the tripledeck structure, where the leading order perturbations equations appear to be degenerate, and in order to deduce the viscous-interaction problem for pressure higher-order terms have to be considered. To make analytic progress, we then assume that the roughness height is small, which is justified physically when considering the vertical extent of 


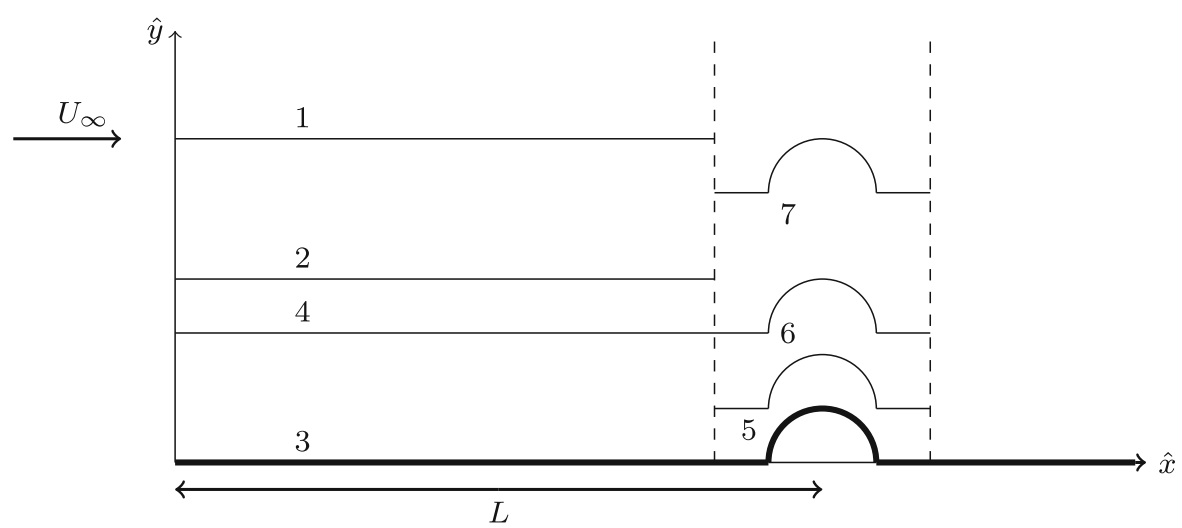

Fig. 1 Schematic of flow regime

roughness elements found on aircraft wings. Solving the resulting linearised equations will enable an expression to be found for the amplitude of the Tollmien-Schlichting waves generated far downstream of the roughness element, at which point the objective of the current paper will have been met.

\section{Problem formulation}

Consider an ideal gas flow over a flat plate aligned with the free stream velocity. We assume that in the flow, there exist small amplitude vorticity waves (uniform free-stream turbulence). In addition, we shall have a roughness element on the flat plate a distant $L$ from the leading edge of said plate. Placing the origin of our coordinate axes at the leading edge of the plate, it is natural to use $(\hat{x}, \hat{y})$ as our coordinates. A schematic of the flow regime can be found in Fig. 1. We shall assume that the flow is two dimensional and that the body force is negligible. We define $(\hat{u}, \hat{v})$ as the velocity vector, $\hat{p}$ the pressure, $\hat{\rho}$ the density, $\hat{h}$ the enthalpy, and $\hat{\mu}$ the viscosity coefficient. We denote the free-stream velocity as $U_{\infty}$, and the free-stream pressure, density, and viscosity coefficient as $p_{\infty}, \rho_{\infty}$, and $\mu_{\infty}$, respectively. In order to non-dimensionalise the compressible Navier-Stokes equations, we introduce the following variables:

$$
\begin{aligned}
& \hat{t}=\frac{L}{U_{\infty}} t, \quad \hat{x}=L x, \quad \hat{y}=L y, \quad \hat{u}=U_{\infty} u, \quad \hat{v}=U_{\infty} v \\
& \hat{\rho}=\rho_{\infty} \rho, \quad \hat{p}=p_{\infty}+\rho_{\infty} U_{\infty}^{2} p, \quad \hat{\mu}=\mu_{\infty} \mu .
\end{aligned}
$$

Substituting these new variables back into our dimensional Navier-Stokes equations gives us the following nondimensional set of equations:

$$
\begin{aligned}
\rho\left(\frac{\partial u}{\partial t}+u \frac{\partial u}{\partial x}+v \frac{\partial u}{\partial y}\right)= & -\frac{\partial p}{\partial x}+\frac{1}{\operatorname{Re}}\left(\frac{\partial}{\partial x}\left[\mu\left(\frac{4}{3} \frac{\partial u}{\partial x}-\frac{2}{3} \frac{\partial v}{\partial y}\right)\right]+\frac{\partial}{\partial y}\left[\mu\left(\frac{\partial u}{\partial y}+\frac{\partial v}{\partial x}\right)\right]\right), \\
\rho\left(\frac{\partial v}{\partial t}+u \frac{\partial v}{\partial x}+v \frac{\partial v}{\partial y}\right)= & -\frac{\partial p}{\partial y}+\frac{1}{\operatorname{Re}}\left(\frac{\partial}{\partial y}\left[\mu\left(\frac{4}{3} \frac{\partial v}{\partial y}-\frac{2}{3} \frac{\partial u}{\partial x}\right)\right]+\frac{\partial}{\partial x}\left[\mu\left(\frac{\partial u}{\partial y}+\frac{\partial v}{\partial x}\right)\right]\right), \\
\rho\left(\frac{\partial h}{\partial t}+u \frac{\partial h}{\partial x}+v \frac{\partial h}{\partial y}\right)= & \frac{\partial p}{\partial t}+u \frac{\partial p}{\partial x}+v \frac{\partial p}{\partial y}+\frac{1}{\operatorname{Re}}\left[\frac{1}{\operatorname{Pr}}\left(\frac{\partial}{\partial x}\left(\mu \frac{\partial h}{\partial x}\right)+\frac{\partial}{\partial y}\left(\mu \frac{\partial h}{\partial y}\right)\right)\right. \\
& \left.+\mu\left(\frac{4}{3} \frac{\partial u}{\partial x}-\frac{2}{3} \frac{\partial v}{\partial y}\right) \frac{\partial u}{\partial x}+\mu\left(\frac{4}{3} \frac{\partial v}{\partial y}-\frac{2}{3} \frac{\partial u}{\partial x}\right) \frac{\partial v}{\partial y}+\mu\left(\frac{\partial u}{\partial y}+\frac{\partial v}{\partial x}\right)^{2}\right],
\end{aligned}
$$


$\frac{\partial \rho}{\partial t}+\frac{\partial(\rho u)}{\partial x}+\frac{\partial(\rho v)}{\partial y}=0$

$h=\frac{1}{(\gamma-1) M_{\infty}^{2}} \frac{1}{\rho}+\frac{\gamma}{\gamma-1} \frac{p}{\rho}$.

Here, we have defined the Reynolds number $(\mathrm{Re})$ and the free-stream Mach number $\left(M_{\infty}\right)$ as follows:

$\operatorname{Re}=\frac{\rho_{\infty} U_{\infty} L}{\mu_{\infty}}, \quad M_{\infty}=\frac{U_{\infty}}{a_{\infty}}$,

respectively, where $a_{\infty}=\sqrt{\left(\gamma p_{\infty}\right) / \rho_{\infty}}$ is the speed of sound in the undisturbed flow upstream of the plate. In the analysis that follows, we will take the limit $\operatorname{Re} \rightarrow \infty$ and keep the Mach number as a constant which is close to 1 .

\section{Flow upstream of the roughness element}

\subsection{Free-stream flow (region 1)}

First, we shall consider the region far away from the plate (and upstream of the roughness). We look for solutions to the Navier-Stokes equations of the form

$$
\begin{aligned}
& u=1+\varepsilon u_{1}(x, y, t), \quad v=\varepsilon v_{1}(x, y, t), \quad \rho=1+\varepsilon \rho_{1}(x, y, t), \\
& p=\varepsilon p_{1}(x, y, t), \quad h=\frac{1}{(\gamma-1) M_{\infty}^{2}}+\varepsilon h_{1}(x, y, t),
\end{aligned}
$$

where $\varepsilon$ is a small parameter. Substituting these asymptotic expansions into the Navier-Stokes equations, we obtain

$$
\begin{aligned}
& \frac{\partial u_{1}}{\partial t}+\frac{\partial u_{1}}{\partial x}=-\frac{\partial p_{1}}{\partial x}, \\
& \frac{\partial v_{1}}{\partial t}+\frac{\partial v_{1}}{\partial x}=-\frac{\partial p_{1}}{\partial y}, \\
& \frac{\partial h_{1}}{\partial t}+\frac{\partial h_{1}}{\partial x}=\frac{\partial p_{1}}{\partial t}+\frac{\partial p_{1}}{\partial x}, \\
& \frac{\partial \rho_{1}}{\partial t}+\frac{\partial \rho_{1}}{\partial x}+\frac{\partial u_{1}}{\partial x}+\frac{\partial v_{1}}{\partial y}=0, \\
& h_{1}=-\frac{1}{(\gamma-1) M_{\infty}^{2}} \rho_{1}+\frac{\gamma}{\gamma-1} p_{1} .
\end{aligned}
$$

It is clear that we can immediately eliminate $h_{1}$ from (3.2c) using (3.2e) giving

$$
\frac{\partial \rho_{1}}{\partial t}+\frac{\partial \rho_{1}}{\partial x}=M_{\infty}^{2}\left(\frac{\partial p_{1}}{\partial t}+\frac{\partial p_{1}}{\partial x}\right)
$$

We can look for wavelike solutions to this equation and (3.2a, 3.2b, 3.2d) of the form $u_{1}(\xi, y), v_{1}(\xi, y), \rho_{1}(\xi, y)$, $p_{1}(\xi, y)$ where $\xi=x-t$. Consequently, we write

$u_{1}=U \mathrm{e}^{\mathrm{i}(\alpha \xi+\beta y)}, \quad v_{1}=V \mathrm{e}^{\mathrm{i}(\alpha \xi+\beta y)}, \quad \rho_{1}=R \mathrm{e}^{\mathrm{i}(\alpha \xi+\beta y)}, \quad p_{1}=P \mathrm{e}^{\mathrm{i}(\alpha \xi+\beta y)}$, 
where $\alpha$ and $\beta$ are the wave numbers in the $x$ and $y$ directions, respectively, and $U, V, P$, and $R$ are constants. Substituting these representations into our equations results in a eigenvalue problem for $c$. In order for us to obtain non-trivial solutions, we require either (i) $U=V=P=0, R$ arbitrary or (ii) $P=R=0$. Vorticity waves correspond to the case where $R=P=0$, so this is the case we shall be concerned with. We will consider the case of one harmonic, so we define vorticity as follows:

$\omega=\frac{\partial v_{1}}{\partial x}-\frac{\partial u_{1}}{\partial y}=\omega_{0} \mathrm{e}^{\mathrm{i}(\alpha \xi+\beta y)}$

where $\omega_{0}$ the amplitude of the wave. Substituting $R=P=0$ into our governing equations, we see that the only equation not automatically satisfied is

$\frac{\partial u_{1}}{\partial x}+\frac{\partial v_{1}}{\partial y}=0$.

Combining this with our definition of vorticity, and without loss of generality setting $\omega_{0}=1$, we obtain the following solutions for the velocity components:

$u_{1}=\frac{\mathrm{i} \beta}{\alpha^{2}+\beta^{2}} \mathrm{e}^{\mathrm{i}(\alpha \xi+\beta y)}$,

$v_{1}=-\frac{\mathrm{i} \alpha}{\alpha^{2}+\beta^{2}} \mathrm{e}^{\mathrm{i}(\alpha \xi+\beta y)}$.

Of course due to Eq. (3.2e), we have that $h_{1} \equiv 0$.

\subsection{Vorticity deformation layer (region 2)}

The work of [10] showed that introducing free-stream turbulence to flow over a flat plate causes a new layer of flow to be formed at $y \sim O\left(\mathrm{Re}^{-1 / 4}\right)$. With that in mind, we begin conducting analysis of this region by introducing the scaled variables:

$\bar{x}=\operatorname{Re}^{-1 / 4} x, \quad \bar{y}=\operatorname{Re}^{-1 / 4} y, \quad \bar{t}=\operatorname{Re}^{-1 / 4} t$.

We look for perturbed solutions to the Euler equations of the form:

$$
\begin{aligned}
& u=1+\varepsilon \bar{u}_{2}(\bar{x}, \bar{y}, \bar{t}), \quad v=\varepsilon \bar{v}_{2}(\bar{x}, \bar{y}, \bar{t}), \quad \rho=1+\varepsilon \bar{\rho}_{2}(\bar{x}, \bar{y}, \bar{t}), \\
& p=\varepsilon \bar{p}_{2}(\bar{x}, \bar{y}, \bar{t}), \quad h=\frac{1}{(\gamma-1) M_{\infty}^{2}}+\varepsilon \bar{h}_{2}(\bar{x}, \bar{y}, \bar{t}) .
\end{aligned}
$$

Eliminating pressure we find that

$$
\frac{\partial}{\partial \bar{t}}\left(\frac{\partial \bar{v}_{2}}{\partial \bar{x}}-\frac{\partial \bar{u}_{2}}{\partial \bar{y}}\right)+\frac{\partial}{\partial \bar{x}}\left(\frac{\partial \bar{v}_{2}}{\partial \bar{x}}-\frac{\partial \bar{u}_{2}}{\partial \bar{y}}\right)=0 .
$$

Using this in conjunction with the definition of vorticity, we can see that vorticity does not change but is merely transported downstream with the mean free-stream velocity. As the vorticity upstream of the plate is given by (3.5), we can write

$$
\frac{\partial \bar{v}_{2}}{\partial \bar{x}}-\frac{\partial \bar{u}_{2}}{\partial \bar{y}}=\mathrm{e}^{\mathrm{i}(\alpha \bar{\xi}+\beta \bar{y})}
$$


where $\bar{\xi}=\bar{x}-\bar{t}$. This equation suggests that $\bar{u}_{2}$ and $\bar{v}_{2}$ are functions of $\bar{\xi}$ and $\bar{y}$ only. Looking for solutions to the Euler equations of this form, we find

$$
\frac{\partial \bar{p}_{2}}{\partial \bar{y}}=0
$$

Taking into account that there are no pressure perturbations in the free stream, we see that $\bar{p}_{2}=0$. Consequently, the energy equation becomes

$$
\frac{\partial \bar{h}_{2}}{\partial \bar{t}}+\frac{\partial \bar{h}_{2}}{\partial \bar{x}}=0
$$

In the previous section, we found that upstream of the leading edge of the plate $h_{1}=0$. By the above equation, we must, therefore, have $\bar{h}_{2} \equiv 0$ in the entire vorticity deformation layer, from which we can deduce that $\bar{\rho}_{2} \equiv 0$. The continuity equation is then reduced to

$\frac{\partial \bar{u}_{2}}{\partial \bar{x}}+\frac{\partial \bar{v}_{2}}{\partial \bar{y}}=0$.

Using this, we can introduce a stream function $\bar{\psi}_{2}$ which is found to be

$\bar{\psi}_{2}=\mathrm{e}^{\mathrm{i} \alpha \bar{\xi}}\left(C \mathrm{e}^{\alpha \bar{y}}+D \mathrm{e}^{-\alpha \bar{y}}\right)+\frac{1}{\alpha^{2}+\beta^{2}} \mathrm{e}^{\mathrm{i}(\alpha \bar{\xi}+\beta \bar{y})}$

where $C$ and $D$ are constants of integration. Therefore, we have

$$
\begin{aligned}
& \bar{u}_{2}=\alpha \mathrm{e}^{\mathrm{i} \alpha \bar{\xi}}\left(C \mathrm{e}^{\alpha \bar{y}}-D \mathrm{e}^{-\alpha \bar{y}}\right)+\frac{\mathrm{i} \beta}{\alpha^{2}+\beta^{2}} \mathrm{e}^{\mathrm{i}(\alpha \bar{\xi}+\beta \bar{y})}, \\
& \bar{v}_{2}=-\mathrm{i} \alpha \mathrm{e}^{\mathrm{i} \alpha \bar{\xi}}\left(C \mathrm{e}^{\alpha \bar{y}}+D \mathrm{e}^{-\alpha \bar{y}}\right)-\frac{\mathrm{i} \alpha}{\alpha^{2}+\beta^{2}} \mathrm{e}^{\mathrm{i}(\alpha \bar{\xi}+\beta \bar{y})} .
\end{aligned}
$$

Clearly, we require $C=0$ otherwise $\bar{u}_{2}$ will grow exponentially with $\bar{y}$. We can calculate $D$ using the impermeability condition at the plate from which we can deduce that

$D=-\frac{1}{\alpha^{2}+\beta^{2}}$.

\subsection{Steady boundary layer flow (region 3)}

When the $y$ coordinate becomes of order $\left(\mathrm{Re}^{-1 / 2}\right)$, a viscous boundary layer forms close to the plate. As there are no leading order pressure perturbations in the vorticity deformation layer, free-stream turbulence does not penetrate into the boundary layer. Therefore, the flow in the boundary layer is steady. Assuming that that $x$ remains an $O$ (1) quantity, and that $Y=\operatorname{Re}^{1 / 2} y$ is an $O(1)$ quantity as $\operatorname{Re} \rightarrow \infty$, we look for solutions to the compressible Navier-Stokes equations of the form:

$$
\begin{array}{ll}
u=U_{0}(x, Y)+\cdots, \quad v=\operatorname{Re}^{-\frac{1}{2}} V_{0}(x, Y)+\cdots, & \rho=\rho_{0}(x, Y)+\cdots, \\
p=\operatorname{Re}^{-\frac{1}{2}} P_{1}(x, Y)+\cdots, \quad h=h_{0}(x, Y)+\cdots, & \mu=\mu_{0}(x, Y)+\cdots
\end{array}
$$

Substituting these representations into the governing equations and letting $\operatorname{Re} \rightarrow \infty$, we obtain (to leading order)

$$
\rho_{0} U_{0} \frac{\partial U_{0}}{\partial x}+\rho_{0} V_{0} \frac{\partial U_{0}}{\partial y}=\frac{\partial}{\partial Y}\left(\mu_{0} \frac{\partial U_{0}}{\partial Y}\right)
$$


$\rho_{0} U_{0} \frac{\partial h_{0}}{\partial x}+\rho_{0} V_{0} \frac{\partial h_{0}}{\partial y}=\frac{1}{\operatorname{Pr}} \frac{\partial}{\partial Y}\left(\mu_{0} \frac{\partial h_{0}}{\partial Y}\right)+\mu_{0}\left(\frac{\partial U_{0}}{\partial Y}\right)^{2}$,

$\frac{\partial}{\partial x}\left(\rho_{0} U_{0}\right)+\frac{\partial}{\partial Y}\left(\rho_{0} V_{0}\right)=0$,

$h_{0}=\frac{1}{(\gamma-1) M_{\infty}^{2}} \frac{1}{\rho_{0}}$.

The boundary conditions of the system are as follows. At the leading edge of the plate, we have

$U_{0}=1, \quad h_{0}=\frac{1}{(\gamma-1) M_{\infty}^{2}}$ at $x=0, Y \in[0, \infty)$.

On the surface of the plate, we have the no-slip and impermeability conditions, which are expressed as follows:

$U_{0}=V_{0}=0$ at $Y=0, x \in[0,1]$.

The matching condition at the outer edge of the boundary is

$U_{0}=1, \quad h_{0}=\frac{1}{(\gamma-1) M_{\infty}^{2}} \quad$ as $\quad Y \rightarrow \infty, x \in[0,1]$.

We will also choose a thermally insulated wall, which has a constant temperature. We write this condition as follows:

$h_{0}=h_{w} \quad$ at $Y=0, x \in[0,1]$.

Self similar solutions to this system of the variable $\eta=Y / \sqrt{x}$ exist. We seek solutions of the form

$U_{0}(x, Y)=U(\eta), \quad V_{0}(x . Y)=\frac{1}{\sqrt{x}} V(\eta), \quad \rho_{0}(x, Y)=\rho(\eta)$,
$h_{0}(x, Y)=h(\eta), \quad \mu_{0}(x, Y)=\mu(\eta)$.

In the limit $\eta \rightarrow \infty$, we have the solutions:

$U=1-\frac{B}{2(\eta+A)} \mathrm{e}^{-\frac{1}{4}(\eta+A)^{2}}, \quad \rho=1, \quad V=-\frac{1}{2} A, \quad h=\frac{1}{(\gamma-1) M_{\infty}^{2}}+\frac{C}{2(\eta+A)} \mathrm{e}^{-\frac{\operatorname{Pr}}{4}(\eta+A)^{2}}$.

where $A, B$, and $C$ are constants.

In the limit $\eta \rightarrow 0$, we consider the flow in the neighbourhood of the roughness element and Taylor expands our solutions in this limit around $x=1$. We find to leading order

$u=\lambda Y, \quad V=0, \quad \rho=\rho_{w}, \quad h=h_{w}, \quad \mu=\mu_{w}$,

where $\lambda$ is the usual Blasius constant.

\subsection{Transition layer (region 4)}

Outside of the boundary layer, we can see that there are no pressure perturbations to leading order. As a consequence, there is no mechanism by which our vorticity waves can penetrate into the boundary layer. However, we cannot 
have a discontinuity in vorticity at the outer edge of the boundary layer. This leads us to introduce a transition region between the classical boundary layer and the vorticity deformation layer, where the vorticity waves decay. We shall seek the solution to the Navier-Stokes equations in this region in the form:

$$
\begin{aligned}
u & =U_{4}(x, Y)+\varepsilon u_{4}(x, \bar{\xi}, \bar{Y})+\cdots, \\
v & =\operatorname{Re}^{-1 / 2} V_{4}(x, Y)+\varepsilon v_{4}(x, \bar{\xi}, \bar{Y})+\cdots, \\
h & =H_{4}(x, Y)+\sigma h_{4}(x, \bar{\xi}, \bar{Y})+\cdots, \\
\rho & =R_{4}(x, Y)+\sigma \rho_{4}(x, \bar{\xi}, \bar{Y})+\cdots, \\
\mu & =\mu(x, Y)+\sigma \mu_{4}(x, \bar{\xi}, \bar{Y})+\cdots,
\end{aligned}
$$

where we assume that the perturbations of pressure are small enough to be neglected. The perturbations of $u$ and $v$ are assumed to be of the same order as those in the vorticity deformation layer, and the order of $\sigma$ is to be found. The coordinates $(x, Y)$ are the same as those in the boundary layer, and $\bar{\xi}$ is as in the vorticity deformation layer. We define $\bar{Y}$ through

$y=\operatorname{Re}^{-1 / 2}[\Delta(\operatorname{Re}) \sqrt{x}-A \sqrt{x}+\delta(\operatorname{Re}) \bar{Y}]$,

where we have that as $\operatorname{Re} \rightarrow \infty, \Delta(\operatorname{Re}) \rightarrow \infty, \delta(\operatorname{Re}) \rightarrow 0$. Rearranging for $\bar{Y}$, we have

$\bar{Y}=\frac{y \operatorname{Re}^{1 / 2}+A \sqrt{x}-\Delta(\operatorname{Re}) \sqrt{x}}{\delta(\operatorname{Re})}$.

Substituting the expansions into the Navier-Stokes equations, we find that

$U_{4}-1=-\frac{B}{2 \Delta} \mathrm{e}^{-\frac{\Delta^{2}}{4}} \mathrm{e}^{-\frac{\Delta \delta \bar{Y}}{2 \sqrt{x}}}$.

If this is constant, then the periodic solution in $\bar{\xi}$ would be a superposition of exponential functions to the power of $\bar{Y}$. This would make it impossible to satisfy both boundary conditions for vorticity at each edge of the transition layer, so in order to prevent this, we set

$$
\Delta \delta=2 .
$$

In what follows we will take our leading order expansions $U_{4}, V_{4}, \rho_{4}$, and $\mu_{4}$ from the outer edge of the Blasius boundary layer studied in the previous section. To avoid degeneration of the first term in the energy equation, we will set

$\operatorname{Re}^{\frac{1}{4}} \frac{B}{2 \Delta} \mathrm{e}^{-\frac{\Delta^{2}}{4}}=\frac{1}{\delta^{2}}$

This reduces our equations to

$$
\begin{aligned}
& -\frac{\sigma}{\delta^{2}} \mathrm{e}^{-\frac{\bar{Y}}{\sqrt{x}}} \frac{\partial \rho_{4}}{\partial \bar{\xi}}+\frac{\varepsilon}{\delta} \operatorname{Re}^{\frac{1}{2}} \frac{\partial v_{4}}{\partial \bar{Y}}-\frac{\varepsilon}{\delta^{2} \sqrt{x}} \frac{\partial u_{4}}{\partial \bar{Y}}-\frac{\sigma}{\delta^{2} \sqrt{x}} \frac{\partial \rho_{4}}{\partial \bar{Y}}=0, \\
& -\frac{\sigma}{\delta^{2}} \mathrm{e}^{-\frac{\bar{Y}}{\sqrt{x}}} \frac{\partial h_{4}}{\partial \bar{\xi}}+\varepsilon v_{4} \operatorname{Re}^{\frac{1}{2}} \frac{\partial h_{4}}{\partial Y}-\frac{\sigma}{\delta^{2} \sqrt{x}} \frac{\partial h_{4}}{\partial \bar{Y}}=\frac{1}{\operatorname{Pr}} \frac{\sigma}{\delta^{2}} \frac{\partial^{2} h_{4}}{\partial \bar{Y}^{2}}+\frac{\varepsilon^{2}}{\delta^{2}}\left[\left(\frac{\partial u_{4}}{\partial \bar{Y}}\right)^{2}+\frac{4}{3}\left(\frac{\partial v_{4}}{\partial \bar{Y}}\right)^{2}\right], \\
& -\frac{\varepsilon}{\delta^{2}} \mathrm{e}^{-\frac{\bar{Y}}{\sqrt{x}}} \frac{\partial u_{4}}{\partial \bar{\xi}}-\frac{\varepsilon}{\delta^{2} \sqrt{x}} \frac{\partial u_{4}}{\partial \bar{Y}}+\varepsilon v_{4} \operatorname{Re}^{\frac{1}{2}} \frac{\partial U_{4}}{\partial \bar{Y}}=\frac{\varepsilon}{\delta^{2}} \frac{\partial^{2} u_{4}}{\partial \bar{Y}^{2}},
\end{aligned}
$$


$h_{4}=-\frac{\rho_{4}}{(\gamma-1) M_{\infty}^{2}}$.

By the principle of least degeneration, we want to keep as many of the terms in these equations as possible. To this end, we set

$\delta=\operatorname{Re}^{-\frac{1}{2}}$

which is consistent with $\delta \rightarrow 0$ as $\operatorname{Re} \rightarrow \infty$, as well setting $\sigma=\varepsilon$. Assuming that the solutions are periodic in $\bar{\xi}$ and matching with the perturbations in both the Blasius boundary layer and vorticity deformation layer, we find

$u_{4}(\bar{\xi}, \bar{Y} ; x)=\mathrm{e}^{\mathrm{i} \alpha \bar{\xi}} \frac{1}{\alpha-\mathrm{i} \beta} J_{0}\left(2 \sqrt{\alpha x \mathrm{i}} \mathrm{e}^{-\frac{\bar{Y}}{2 \sqrt{x}}}\right), \quad h_{4} \equiv 0, \quad \rho_{4} \equiv 0$,

where $J_{0}$ is the zeroth-order Bessel function of the first kind.

\section{Flow in the interaction region}

In order to analyse the flow around the roughness element, we shall use the same triple-deck structure as $[8,9]$. We require a time scale of $t=O\left(\mathrm{Re}^{-2 / 9}\right)$ in order for the resonance conditions with Tollmien-Schlichting waves to be satisfied, and we shall further assume that the Mach number is of the form:

$M_{\infty}=1+\operatorname{Re}^{-1 / 9} Q_{\infty}$

where $Q_{\infty}$ is the Kármán-Guderley parameter.

\subsection{Lower deck (region 5)}

The horizontal extent of the roughness element is assumed to be of the order $x=O\left(\mathrm{Re}^{-1 / 3}\right)$, and the vertical extent of the lower deck is $y=O\left(\mathrm{Re}^{-11 / 18}\right)$. We shall, therefore, assume that the shape of the roughness is of the form:

$y=\operatorname{Re}^{-11 / 18} F\left(\frac{x-1}{\operatorname{Re}^{-1 / 3}}\right)$.

It is natural to introduce re-scaled coordinates $\left(x_{*}, y_{5}\right)$ and time $t_{*}$ as follows:

$x=1+\operatorname{Re}^{-1 / 3} x_{*}, \quad y=\operatorname{Re}^{-11 / 18} y_{5}, \quad t=\operatorname{Re}^{-2 / 9} t_{*}$.

We seek solutions to the Navier-Stokes equations in this region in the form:

$$
\begin{aligned}
u & =\operatorname{Re}^{-\frac{1}{9}} u_{5}\left(x_{*}, y_{5}\right)+\cdots+\varepsilon \tilde{u}_{5}\left(x_{*}, y_{5}, t_{*}\right)+\cdots, \\
v & =\operatorname{Re}^{-\frac{7}{18}} v_{5}\left(x_{*}, y_{5}\right)+\cdots+\varepsilon \operatorname{Re}^{-\frac{5}{18}} \tilde{v}_{5}\left(x_{*}, y_{5}, t_{*}\right)+\cdots, \\
p & =\operatorname{Re}^{-\frac{2}{9}} p_{5}\left(x_{*}, y_{5}\right)+\cdots+\varepsilon \operatorname{Re}^{-\frac{1}{9}} \tilde{p}_{5}\left(x_{*}, y_{5}, t_{*}\right)+\cdots, \\
h & =h_{w}+\cdots, \\
\rho & =\rho_{w}+\cdots, \\
\mu & =\mu_{w}+\cdots,
\end{aligned}
$$


where we assume that close to the roughness element enthalpy, viscosity, and density are constants, and $\varepsilon<<\mathrm{Re}^{-1 / 9}$ to prevent the expansion becoming disordered. The first terms in these expansions correspond to the steady flow solution, whereas those that are $\mathrm{O}(\varepsilon)$ are due to time-dependent perturbations. Substituting these expansions into the non-dimensional Navier-Stokes equations yields to $\mathrm{O}(1)$ :

$$
\begin{aligned}
& \rho_{w}\left(u_{5} \frac{\partial u_{5}}{\partial x_{*}}+v_{5} \frac{\partial u_{5}}{\partial y_{5}}\right)=-\frac{\partial p_{5}}{\partial x_{*}}+\mu_{w} \frac{\partial^{2} u_{5}}{\partial y_{5}^{2}}, \\
& \frac{\partial p_{5}}{\partial y_{5}}=0, \\
& \frac{\partial u_{5}}{\partial x_{*}}+\frac{\partial v_{5}}{\partial y_{5}}=0 .
\end{aligned}
$$

Our boundary conditions for these equations come from our no-slip conditions on the roughness element, and matching $u$ with flow upstream of the roughness as $x_{*} \rightarrow-\infty$. These are

$u_{5}=v_{5}=0 \quad$ at $y_{5}=F\left(x_{*}\right)$,

$u_{5}=\lambda y_{5} \quad$ as $\quad x_{*} \rightarrow-\infty$.

Clearly, we can see that the pressure $p_{5}$ does not change across the lower deck with respect to the $y$-direction. Using the continuity equation we find that in the limit $y_{5} \rightarrow \infty$

$u_{5}=\lambda y_{5}+A_{*}, \quad v_{5}=-\frac{\mathrm{d} A_{*}}{\mathrm{~d} x_{*}} y_{5}$,

where the function $A_{*}$ is still to be found. We can perform a similar analysis on the $\mathrm{O}(\varepsilon)$ terms in our expansions. The boundary conditions for this new problem are

$$
\begin{aligned}
& \tilde{u}_{5}=\tilde{v}_{5}=0 \text { at } y_{5}=F\left(x_{*}\right), \\
& \tilde{u}_{5}=0 \text { as } x_{*} \rightarrow-\infty .
\end{aligned}
$$

Substituting into the Navier-Stokes equations, we obtain the $\mathrm{O}(\varepsilon)$ equations:

$$
\begin{aligned}
& \rho_{w}\left(\frac{\partial \tilde{u}_{5}}{\partial t_{*}}+u_{5} \frac{\partial \tilde{u}_{5}}{\partial x_{*}}+\tilde{u}_{5} \frac{\partial u_{5}}{\partial x_{*}}+v_{5} \frac{\partial \tilde{u}_{5}}{\partial y_{5}}+\tilde{v}_{5} \frac{\partial u_{5}}{\partial y_{5}}\right)=-\frac{\partial \tilde{p}_{5}}{\partial x_{*}}+\mu_{w} \frac{\partial^{2} \tilde{u}_{5}}{\partial y_{5}^{2}}, \\
& \frac{\partial \tilde{p}_{5}}{\partial y_{5}}=0 \\
& \frac{\partial \tilde{u}_{5}}{\partial x_{*}}+\frac{\partial \tilde{v}_{5}}{\partial y_{5}}=0 .
\end{aligned}
$$

In this case, we find that as $y_{5} \rightarrow \infty$

$$
\tilde{u}_{5}=\tilde{A}_{*}\left(x_{*}, t_{*}\right), \quad \tilde{v}_{5}=-\frac{\partial \tilde{A}_{*}}{\partial x_{*}} y_{5} .
$$


In order to understand, the physical meanings of $A_{*}$ and $\tilde{A}_{*}$ consider the streamline slope angle $\theta$ at the outer edge of region 5. Taking the limit as $y_{5} \rightarrow \infty$, we have

$\theta=\tan ^{-1} \frac{v}{u}=\tan ^{-1}\left(-\frac{\operatorname{Re}^{-\frac{7}{18} \frac{\mathrm{d} A_{*}}{\mathrm{~d} x_{*}} y_{5}+\varepsilon \operatorname{Re}^{-\frac{5}{18}} \frac{\partial \tilde{A}_{*}}{\partial x_{*}} y_{5}}}{\operatorname{Re}^{-\frac{1}{9}}\left(\lambda y_{5}+A_{*}\right)+\varepsilon \tilde{A}_{*}}\right) \approx-\frac{1}{\lambda}\left(\operatorname{Re}^{-\frac{5}{18}} \frac{\mathrm{d} A_{*}}{\mathrm{~d} x_{*}}+\varepsilon \operatorname{Re}^{-\frac{1}{6}} \frac{\partial \tilde{A}_{*}}{\partial x_{*}}\right)$.

Due to this relationship between $\theta, A_{*}$, and $\tilde{A}_{*}$ we call $A_{*}$ the $O(1)$ displacement function and $\tilde{A}_{*}$ the $\mathrm{O}(\varepsilon)$ displacement function.

\subsection{Middle deck (region 6)}

The middle deck region is a continuation of the boundary layer upstream of the roughness. As a consequence, it has vertical extent $y=O\left(\mathrm{Re}^{-1 / 2}\right)$ which leads us to re-scale our $y$-coordinate as follows:

$y=\operatorname{Re}^{-\frac{1}{2}} y_{6}$.

Relating this to the solution for the lower deck by considering the matching conditions for $u$ and $v$ as $y_{6} \rightarrow 0$, we can see that in this limit the solution in the middle deck is

$u=\lambda y_{6}+\operatorname{Re}^{-\frac{1}{9}} A_{*}\left(x_{*}\right)+\cdots+\varepsilon \tilde{A}_{*}\left(x_{*}, t_{*}\right)+\cdots$
$v=\operatorname{Re}^{-\frac{5}{18}} y_{6} \frac{\mathrm{d} A_{*}}{\mathrm{~d} x_{*}}+\cdots+\varepsilon \operatorname{Re}^{-\frac{1}{6}} y_{6} \frac{\partial \tilde{A}_{*}}{\partial x_{*}}+\cdots$

Consequently, we shall seek solutions in this region of the form:

$$
\begin{aligned}
u & =u_{6}\left(y_{6}\right)+\operatorname{Re}^{-\frac{1}{9}} u_{6}^{*}\left(x_{*}, y_{6}\right)+\cdots+\varepsilon \tilde{u}_{6}\left(x_{*}, y_{6}, t_{*}\right)+\cdots, \\
v & =\operatorname{Re}^{-\frac{5}{18}} v_{6}\left(x_{*}, y_{6}\right)+\cdots \varepsilon \operatorname{Re}^{-\frac{1}{6}} \tilde{v}_{6}\left(x_{*}, y_{6}, t_{*}\right)+\cdots, \\
p & =\operatorname{Re}^{-\frac{2}{9}} p_{6}\left(x_{*}, y_{6}\right)+\cdots \varepsilon \operatorname{Re}^{-\frac{1}{9}} \tilde{p}_{6}\left(x_{*}, y_{6}, t_{*}\right)+\cdots, \\
\rho & =\rho_{60}\left(y_{6}\right)+\operatorname{Re}^{-\frac{1}{9}} \rho_{61}\left(x_{*}, y_{6}\right)+\cdots+\varepsilon \tilde{\rho}_{6}\left(x_{*}, y_{6}, t_{*}\right)+\cdots, \\
h & =h_{60}\left(y_{6}\right)+\operatorname{Re}^{-\frac{1}{9}} h_{61}\left(x_{*}, y_{6}\right)+\cdots+\varepsilon \tilde{h}_{6}\left(x_{*}, y_{6}, t_{*}\right)+\cdots, \\
\mu & =\mu_{60}\left(y_{6}\right)+\operatorname{Re}^{-\frac{1}{9}} \mu_{61}\left(x_{*}, y_{6}\right)+\cdots+\varepsilon \tilde{\mu}_{6}\left(x_{*}, y_{6}, t_{*}\right)+\cdots .
\end{aligned}
$$

Substituting these expansions into the Navier-Stokes equations and using elimination to find $u$ and $v$, we find the streamline slope angle to be

$\theta=\operatorname{Re}^{-\frac{5}{18}} K\left(x_{*}\right)+\varepsilon \operatorname{Re}^{-\frac{1}{6}} \tilde{K}\left(x_{*}, t_{*}\right)$,

where $K\left(x_{*}\right), \tilde{K}\left(x_{*}, t_{*}\right)$ are unknown functions. As $\theta$ has no dependence on $y_{6}$ we can match this with streamline slope angle from the lower deck yielding

$$
\theta=-\frac{1}{\lambda}\left(\operatorname{Re}^{-\frac{5}{18}} \frac{\mathrm{d} A_{*}}{\mathrm{~d} x_{*}}+\varepsilon \operatorname{Re}^{-\frac{1}{6}} \frac{\partial \tilde{A}_{*}}{\partial x_{*}}\right)
$$




\subsection{Upper deck (region 7)}

In order to analyse the upper deck, we are required to re-scale $y$ again. As we have that the vertical extent of the upper deck is $y=O\left(\mathrm{Re}^{-5 / 18}\right)$, we shall introduce a new variable $y_{7}$ defined as follows:

$y=\operatorname{Re}^{-\frac{5}{18}} y_{7}$.

We then look for solutions to the Navier-Stokes equations in this region of the form:

$$
\begin{aligned}
u & =1+\operatorname{Re}^{-2 / 9} u_{s t}\left(x_{*}, y_{7}\right) \cdots+\varepsilon u_{\alpha}\left(t_{*}\right)+\cdots+\varepsilon \operatorname{Re}^{-1 / 9} u_{*}\left(t_{*}, x_{*}, y_{7}\right)+\varepsilon \operatorname{Re}^{-2 / 9} \tilde{u}_{*}\left(t_{*}, x_{*}, y_{7}\right)+\cdots, \\
v & =\operatorname{Re}^{-5 / 18} v_{s t}\left(x_{*}, y_{7}\right)+\cdots+\varepsilon \operatorname{Re}^{-1 / 6} v_{*}\left(t_{*}, x_{*}, y_{7}\right)+\varepsilon \operatorname{Re}^{-5 / 18} \tilde{v}_{*}\left(t_{*}, x_{*}, y_{7}\right)+\cdots, \\
p & =\operatorname{Re}^{-2 / 9} p_{s t}\left(x_{*}, y_{7}\right)+\cdots+\varepsilon e^{-1 / 9} p_{*}\left(t_{*}, x_{*}, y_{7}\right)+\varepsilon \operatorname{Re}^{-2 / 9} \tilde{p}_{*}\left(t_{*}, x_{*}, y_{7}\right)+\cdots, \\
\rho & =1+\operatorname{Re}^{-2 / 9} \rho_{s t}\left(x_{*}, y_{7}\right)+\cdots+\varepsilon \operatorname{Re}^{-1 / 9} \rho_{*}\left(t_{*}, x_{*}, y_{7}\right)+\varepsilon \operatorname{Re}^{-2 / 9} \tilde{\rho}_{*}\left(t_{*}, x_{*}, y_{7}\right)+\cdots, \\
h & =\frac{1}{(\gamma-1) M_{\infty}^{2}}+\operatorname{Re}^{-2 / 9} h_{s t}\left(x_{*}, y_{7}\right)+\cdots+\varepsilon \operatorname{Re}^{-1 / 9} h_{*}\left(t_{*}, x_{*}, y_{7}\right)+\varepsilon \operatorname{Re}^{-2 / 9} \tilde{h}_{*}\left(t_{*}, x_{*}, y_{*}\right)+\cdots,
\end{aligned}
$$

where $u_{\alpha}$ is a time-dependent perturbation due to vorticity which is known to be of the form $e^{i \alpha t_{*}}$, and $u_{s t}, v_{s t}, p_{s t}$, $\rho_{s t}$, and $h_{s t}$ are the solutions in the steady flow. Derivatives of $u_{\alpha}$ will be cancelled out by next order expansions of the perturbation terms, which have been omitted in the asymptotic expansion of $u$. Substituting these expansions into the Navier-Stokes equations, we obtain to $O(1)$ :

$$
\begin{aligned}
& \frac{\partial u_{s t}}{\partial x_{*}}=-\frac{\partial p_{s t}}{\partial x_{*}}, \\
& \frac{\partial v_{s t}}{\partial x_{*}}=-\frac{\partial p_{s t}}{\partial y_{7}}, \\
& \frac{\partial \rho_{s t}}{\partial x_{*}}=\frac{\partial p_{s t}}{\partial x_{*}}, \\
& \frac{\partial u_{s t}}{\partial x_{*}}+\frac{\partial \rho_{s t}}{\partial x_{*}}=0 .
\end{aligned}
$$

Using (4.19c) in (4.19d) and comparing this to (4.19a), we can see that this system is degenerate. It is, therefore, necessary to retain the next order terms, from which we obtain the equation

$2 Q_{\infty} \frac{\partial p_{s t}}{\partial x_{*}}+\frac{\partial v_{s t}}{\partial y_{7}}=0$

We, therefore, have that the governing equation for $p_{s t}$ is given by

$2 Q_{\infty} \frac{\partial^{2} p_{s t}}{\partial x_{*}^{2}}-\frac{\partial^{2} p_{s t}}{\partial y_{7}^{2}}=0$.

This is accompanied by the boundary condition that the perturbations are expected to decay far away from the roughness

$u_{s t}, v_{s t}, p_{s t}, \rho_{s t} \rightarrow 0 \quad$ as $\quad x_{*}^{2}+y_{7}^{2} \rightarrow \infty$ 
We now turn to the unsteady terms in our expansions (4.18). Our $\mathrm{O}(\varepsilon)$ equations are

$$
\begin{aligned}
& \frac{\partial u_{*}}{\partial x_{*}}=-\frac{\partial p_{*}}{\partial x_{*}}, \\
& \frac{\partial v_{*}}{\partial x_{*}}=-\frac{\partial p_{*}}{\partial y_{7}}, \\
& \frac{\partial p_{*}}{\partial x_{*}}-\frac{\partial \rho_{*}}{\partial x_{*}}=0, \\
& \frac{\partial u_{*}}{\partial x_{*}}+\frac{\partial \rho_{*}}{\partial x_{*}}=0 .
\end{aligned}
$$

This system is again degenerate, so it is necessary to consider the next order equations which are

$u_{\alpha} \frac{\partial u_{s t}}{\partial x_{*}}+\frac{\partial u_{*}}{\partial t_{*}}+\frac{\partial \tilde{u}_{*}}{\partial x_{*}}=-\frac{\partial \tilde{p}_{*}}{\partial x_{*}}$,

$u_{\alpha} \frac{\partial v_{s t}}{\partial x_{*}}+\frac{\partial v_{*}}{\partial t_{*}}+\frac{\partial \tilde{v_{*}}}{\partial x_{*}}=-\frac{\partial \tilde{p}_{*}}{\partial y_{7}}$,

$u_{\alpha} \frac{\partial p_{s t}}{\partial x_{*}}-u_{\alpha} \frac{\partial \rho_{s t}}{\partial x_{*}}+\frac{\partial p_{*}}{\partial t_{*}}-\frac{\partial \rho_{*}}{\partial t_{*}}+\frac{\partial \tilde{p}_{*}}{\partial x_{*}}-\frac{\partial \tilde{\rho}_{*}}{\partial x_{*}}+2 Q_{\infty} \frac{\partial \rho_{*}}{\partial x_{*}}+2 Q_{\infty} u_{\alpha} \frac{\partial \rho_{s t}}{\partial x_{*}}=0$,

$u_{\alpha} \frac{\partial \rho_{s t}}{\partial x_{*}}+\frac{\partial \rho_{*}}{\partial t_{*}}+\frac{\partial \tilde{\rho}_{*}}{\partial x_{*}}+\frac{\partial \tilde{u_{*}}}{\partial x_{*}}+\frac{\partial v_{*}}{\partial y_{7}}=0$.

Making use of Eqs. (4.19) and (4.23), this problem can be reduced to the following equation for the unsteady pressure perturbation in the upper tier

$2 Q_{\infty} \frac{\partial^{2} p_{*}}{\partial x_{*}^{2}}+2 \frac{\partial^{2} p_{*}}{\partial t_{*} \partial x_{*}}-\frac{\partial^{2} p_{*}}{\partial y_{7}^{2}}=-2 u_{\alpha} \frac{\partial^{2} p_{s t}}{\partial x_{*}^{2}}$

and of course, we expect a similar decay of these perturbations as in the $\mathrm{O}(1)$ case.

In order to close our viscous-inviscid interaction problem for $p_{*}$, we require an additional boundary condition at $y_{7}=0$ for both steady and unsteady flow. To do this, we consider our streamline slope angle $\theta$ for region 6 . So far, we have not considered the continuation of our transition layer into the triple-deck model. However, using a similar argument to [12], we can see that this is not necessary. The displacement thickness of the boundary layer has only been affected by the viscous lower deck, where the velocity was of small magnitude in comparison with other regions. We expect the velocity to be continuous across the transition layer of the triple deck, so it would be natural for it to be of the same order as the velocity in regions 6 and 7. Therefore, we can reasonably assume that the streamline slope angle will not be affected by this layer, as it is unaffected by region 6 which has the same magnitude of velocity and is much thicker. Consequently, the streamline slope angle at the bottom of region 7 is the same as it is in region 6 so we have

$\theta=-\operatorname{Re}^{-\frac{5}{18}} \frac{1}{\lambda}\left(\operatorname{Re}^{-\frac{5}{18}} \frac{\mathrm{d} A_{*}}{\mathrm{~d} x_{*}}+\varepsilon \operatorname{Re}^{-\frac{1}{6}} \frac{\partial \tilde{A}_{*}}{\partial x_{*}}\right) \quad$ at $\quad y_{7}=0$.

From this, we can derive the boundary condition on $p_{s t}$ and $p_{*}$ at $y_{7}=0$ which are

$\frac{\partial p_{s t}}{\partial y_{7}}=\frac{1}{\lambda} \frac{\mathrm{d}^{2} A_{*}}{\partial x_{*}^{2}}$, 
and $\frac{\partial p_{*}}{\partial y_{7}}=\frac{1}{\lambda} \frac{\partial^{2} \tilde{A}_{*}}{\partial x_{*}^{2}} \quad$ at $\quad y_{7}=0$,

respectively. For steady flow, we, therefore, have

$2 Q_{\infty} \frac{\partial^{2} p_{s t}}{\partial x_{*}^{2}}-\frac{\partial^{2} p_{s t}}{\partial y_{7}^{2}}=0$,
$p_{s t} \rightarrow 0$ as $x_{*}^{2}+y_{7}^{2} \rightarrow \infty$,
$\frac{\partial p_{s t}}{\partial y_{7}}=\frac{1}{\lambda} \frac{\mathrm{d}^{2} A_{*}}{\mathrm{~d} x_{*}^{2}}$ at $y_{7}=0$.

For unsteady perturbations, we have

$$
\begin{aligned}
& 2 Q_{\infty} \frac{\partial^{2} p_{*}}{\partial x_{*}^{2}}+2 \frac{\partial^{2} p_{*}}{\partial t_{*} \partial x_{*}}-\frac{\partial^{2} p_{*}}{\partial y_{7}^{2}}=-2 u_{\alpha} \frac{\partial^{2} p_{s t}}{\partial x_{*}^{2}} . \\
& p_{*} \rightarrow 0 \text { as } x_{*}^{2}+y_{7}^{2} \rightarrow \infty, \\
& \frac{\partial p_{*}}{\partial y_{7}}=\frac{1}{\lambda} \frac{\partial^{2} \tilde{A}_{*}}{\partial x_{*}^{2}} \text { at } y_{7}=0 .
\end{aligned}
$$

\section{Linear receptivity}

In order to make analytic progress with our systems of equations (both steady and non-steady), we assume that the height of the roughness is small in comparison to the lower deck. We, therefore, write the equation of the roughness to be

$y=\delta f(x)$ where $\delta \ll 1$.

Using this assumption, we can linearise our equations for the lower deck. In this region, we, therefore, write the expansions for our velocities terms and pressure as follows:

$$
\begin{aligned}
u & =\lambda y_{5}+\delta u_{5}^{\prime}\left(x_{*}, y_{5}\right)+\cdots+\delta \sigma \tilde{u}_{5}^{\prime}\left(x_{*}, y_{5}, t_{*}\right)+\cdots, \\
v & =\delta v_{5}^{\prime}\left(x_{*}, y_{5}\right)+\cdots+\delta \sigma \tilde{v}_{5}^{\prime}\left(x_{*}, y_{5}, t_{*}\right)+\cdots, \\
p & =h P_{5}^{\prime}\left(x_{*}, y_{5}\right)+\cdots+\delta \sigma \tilde{P}_{5}^{\prime}\left(x_{*}, y_{5}, t_{*}\right)+\cdots
\end{aligned}
$$

where $\sigma$ is a small parameter. We shall also perform similar expansions for the displacement function $A$, and upper deck pressure $p$ of the form:

$$
\begin{aligned}
A & =\delta A_{*}^{\prime}\left(x_{*}\right)+\cdots+\delta \sigma \tilde{A}_{*}^{\prime}\left(x_{*}, t_{*}\right)+\cdots, \\
p & =\delta p_{s t}^{\prime}\left(x_{*}, y_{7}\right)+\cdots+\delta \sigma p_{*}^{\prime}\left(x_{*}, t_{*}\right)+\cdots .
\end{aligned}
$$

We now substitute these representations into our equations for the lower deck. First, we shall consider the problem for steady flow. 


\subsection{Steady problem}

Our linearised equations for steady flow to $O(\delta)$ read

$\rho_{w}\left(\lambda y_{5} \frac{\partial u_{5}^{\prime}}{\partial x_{*}}+\lambda v_{5}^{\prime}\right)=-\frac{\partial P_{5}^{\prime}}{\partial x_{*}}+\mu_{w} \frac{\partial^{2} u_{5}^{\prime}}{\partial y_{5}^{2}}$,

$\frac{\partial P_{5}^{\prime}}{\partial y_{5}}=0$,

$\frac{\partial u_{5}^{\prime}}{\partial x_{*}}+\frac{\partial v_{5}^{\prime}}{\partial y_{5}}=0$.

We also have the boundary conditions:

$u_{5}^{\prime}=-\lambda f\left(x_{*}\right)$ at $y_{5}=0$,

$v_{5}^{\prime}=0$ at $y_{5}=0$,

$u_{5}^{\prime}=A_{*}^{\prime} \quad$ as $y_{5} \rightarrow \infty$,

$u_{5}^{\prime}=0 \quad$ as $\quad x_{*} \rightarrow-\infty$.

We also need to relate $P_{5}^{\prime}$ to $p_{s t}$. As the pressure distribution does not change across the middle deck, we can relate $P_{5}^{\prime}$ back to the pressure in the lower deck by the equation:

$\left.p_{s t}\right|_{y_{7}=0}=P_{5}^{\prime}$.

This system of equations will be solved in the usual way of transforming our variables into Fourier space. We define the Fourier transforms as follows:

$\left(\bar{P}_{5}, \bar{p}_{s t}\right)=\int_{-\infty}^{\infty} \mathrm{e}^{-\mathrm{i} k x_{*}}\left(P_{5}^{\prime}, p_{s t}\right) \mathrm{d} x_{*}$.

Defining a new variable $z=(\mathrm{i} k)^{\frac{1}{3}} y_{5}$, we find the solution for subsonic flow $\left(Q_{\infty}<0\right)$ to be

$\begin{aligned} \bar{P}_{5} & =\frac{|k|}{\sqrt{2\left|Q_{\infty}\right|}} \frac{\bar{f}(k) q^{\frac{2}{3}} A i^{\prime}(0)}{q^{\frac{2}{3}} A i^{\prime}(0)-\frac{(i k)^{\frac{1}{3}}|k|}{\lambda \mu_{w} \sqrt{2\left|Q_{\infty}\right|}} \int_{0}^{\infty} A i\left(q^{\frac{2}{3}} z^{\prime}\right) \mathrm{d} z^{\prime}}, \\ \bar{p}_{s t} & =\frac{|k|}{\sqrt{2\left|Q_{\infty}\right|}} \frac{\bar{f}(k) q^{\frac{2}{3}} A i^{\prime}(0)}{q^{\frac{2}{3}} A i^{\prime}(0)-\frac{(i k)^{\frac{1}{3}}|k|}{\lambda \mu_{w} \sqrt{2\left|Q_{\infty}\right|}} \int_{0}^{\infty} A i\left(q^{\frac{2}{3}} z^{\prime}\right) \mathrm{d} z^{\prime}} \mathrm{e}^{-|k| \sqrt{2\left|Q_{\infty}\right|} y_{7}},\end{aligned}$

where $\bar{p}_{s t}$ is the Fourier transform of $p_{s t}$, and $\bar{f}$ is the Fourier transform of the roughness shape. To find the solution for supersonic flow $\left(Q_{\infty}>0\right)$, we have to take into account the causality condition, which yields the solutions

$$
\begin{aligned}
\bar{P}_{5} & =\frac{\mathrm{i} k}{\sqrt{2 Q_{\infty}}} \frac{\bar{f}(k) q^{\frac{2}{3}} A i^{\prime}(0)}{q^{\frac{2}{3}} A i^{\prime}(0)+\mathrm{i} \frac{(\mathrm{i} k)^{\frac{1}{3}} k}{\lambda \mu_{w} \sqrt{2 Q_{\infty}}} \int_{0}^{\infty} A i\left(q^{\frac{2}{3}} z^{\prime}\right) \mathrm{d} z^{\prime}}, \\
\bar{p}_{s t} & =\frac{i k}{\sqrt{2 Q_{\infty}}} \frac{\bar{f}(k) q^{\frac{2}{3}} A i^{\prime}(0)}{q^{\frac{2}{3}} A i^{\prime}(0)+\mathrm{i} \frac{(\mathrm{i} k)^{\frac{1}{3}} k}{\lambda \mu_{w} \sqrt{2 Q_{\infty}}} \int_{0}^{\infty} A i\left(q^{\frac{2}{3}} z^{\prime}\right) \mathrm{d} z^{\prime}} \mathrm{e}^{-\mathrm{ik} \sqrt{2 Q_{\infty}} y_{7}} .
\end{aligned}
$$


At this point, we note that the branch cut of $(\mathrm{i} k)^{\frac{1}{3}}$ in the complex $k$-plane is chosen in the usual way to be the positive imaginary semi-axis.

\subsection{Unsteady problem}

In order to solve our unsteady problem, we consider the terms of $O(\sigma \delta)$ in our linearised unsteady lower deck equations which give

$\rho_{w}\left(\frac{\partial \tilde{u}_{5}^{\prime}}{\partial t_{*}}+\lambda y_{5} \frac{\partial \tilde{u}_{5}^{\prime}}{\partial x_{*}}+\lambda \tilde{v}_{5}^{\prime}\right)=-\frac{\partial \tilde{P}_{5}^{\prime}}{\partial x_{*}}+\mu_{w} \frac{\partial^{2} \tilde{u}_{5}^{\prime}}{\partial y_{7}^{2}}$,

$\frac{\partial \tilde{P}_{5}^{\prime}}{\partial y_{5}}=0$

$\frac{\partial \tilde{u}_{5}^{\prime}}{\partial x_{*}}+\frac{\partial \tilde{v}_{5}^{\prime}}{\partial y_{5}}=0$.

The boundary conditions for this problem are

$\tilde{u}_{5}^{\prime}=\tilde{v}_{5}^{\prime}=0$ at $y_{5}=0$,

$\tilde{u}_{5}^{\prime}=\tilde{A}_{*}^{\prime}$ as $y_{5} \rightarrow \infty$,

$\tilde{u}_{5}^{\prime}=0 \quad$ as $\quad x_{*} \rightarrow-\infty$.

In order to make analytical progress in finding solutions to these equations, we shall assume that our solutions are periodic in time, with wavenumber $\alpha$ corresponding to the wavenumber of $u_{\alpha}(t)$. This means we can write them in the form:

$$
\left(\tilde{u}_{5}^{\prime}, \tilde{v}_{5}^{\prime}, \tilde{P}_{5}^{\prime}, p_{*}^{\prime}, \tilde{A}_{*}^{\prime}\right)=\mathrm{e}^{\mathrm{i} \alpha t}\left(u_{u}, v_{u}, P_{u}, p_{u}, A_{u}\right)+\mathrm{c} \cdot \mathrm{c}
$$

where the functions on the right-hand side have no time dependence, and c.c denotes the complex conjugate. Substituting these expressions into (5.10) and (5.11) yields

$\rho_{w}\left(\mathrm{i} \alpha u_{u}+\lambda y_{5} \frac{\partial u_{u}}{\partial x_{*}}+\lambda v_{u}\right)=-\frac{\partial P_{u}}{\partial x_{*}}+\mu_{w} \frac{\partial^{2} u_{u}}{\partial y_{7}^{2}}$,

$\frac{\partial P_{u}}{\partial y_{5}}=0$,

$\frac{\partial u_{u}}{\partial x_{*}}+\frac{\partial v_{u}}{\partial y_{5}}=0$

$u_{u}=v_{u}=0$ at $y_{5}=0$,

$u_{u}=A_{u} \quad$ as $\quad y_{5} \rightarrow \infty$,

$u_{u}=0 \quad$ as $\quad x_{*} \rightarrow-\infty$.

Performing the same substitution in the boundary value problem (4.29) derived for the pressure in the upper deck $p_{*}$ gives

$2 \mathrm{i} \alpha \frac{\partial p_{u}}{\partial x_{*}}+2 Q_{\infty} \frac{\partial^{2} p_{u}}{\partial x_{*}^{2}}-\frac{\partial^{2} p_{u}}{\partial y_{7}^{2}}=-2 \frac{\partial^{2} p_{s t}}{\partial x_{*}^{2}}$, 
$p_{u} \rightarrow 0 \quad$ as $\quad x_{*}^{2}+y_{7}^{2} \rightarrow \infty$,

$\frac{\partial p_{u}}{\partial y_{7}}=\frac{1}{\lambda} \frac{\mathrm{d}^{2} A_{u}}{\mathrm{~d} x_{*}^{2}} \quad$ at $\quad y_{7}=0$.

In similar fashion to how we approached the steady problem, we begin by Fourier transforming our variables in $x_{*}$, so that the above problem for $p_{u}$ becomes

$\frac{\mathrm{d}^{2} \bar{p}_{u}}{\mathrm{~d} y_{7}^{2}}+\bar{p}_{u}\left(2 k \alpha+2 Q_{\infty} k^{2}\right)=-2 k^{2} \bar{p}_{s t}$,

$\bar{p}_{u} \rightarrow 0$ as $y_{7} \rightarrow \infty$,

$\frac{\mathrm{d} \bar{p}_{u}}{\mathrm{~d} y_{7}}=-\frac{k^{2}}{\lambda} \bar{A}_{u} \quad$ at $\quad y_{7}=0$,

where $\bar{p}_{u}$ and $\bar{A}_{u}$ are the Fourier transforms of $p_{u}$ and $A_{u}$, respectively, and $\bar{p}_{s t}$ was found in our analysis of the steady problem. The solution to this problem of course depends on the nature of flow (i.e. whether it is subsonic or supersonic) due to the fact that the forcing on the right-hand side of (5.15a) depends on the sign of $Q_{\infty}$. In addition, as we need to use an inverse Fourier transform to return to physical space, we require a solution for all $k$. Therefore, for each sign of $Q_{\infty}$, we have to split our solution into 3 different regions. For $Q_{\infty}<0$, we have

1. $k<0, \quad 2 k \alpha+2 Q_{\infty} k^{2}<0$;

2. $k \in\left(0,-\frac{\alpha}{Q_{\infty}}\right), \quad 2 k \alpha+2 Q_{\infty} k^{2}>0$;

3. $k>-\frac{\alpha}{Q_{\infty}}, \quad 2 k \alpha+2 Q_{\infty} k^{2}<0$.

For $Q_{\infty}>0$, we have

1. $k<-\frac{\alpha}{Q_{\infty}}, \quad 2 k \alpha+2 Q_{\infty} k^{2}>0$;

2. $k \in\left(-\frac{\alpha}{Q_{\infty}}, 0\right), \quad 2 k \alpha+2 Q_{\infty} k^{2}<0$;

3. $k>0, \quad 2 k \alpha+2 Q_{\infty} k^{2}>0$.

We begin by considering the case of subsonic flow.

\subsubsection{Subsonic flow}

In the case of subsonic flow $\left(Q_{\infty}<0\right)$, the solution to the system (5.15) is

$\bar{p}_{u}=\frac{\frac{k^{2}}{\lambda} \bar{A}_{u}+\frac{k}{\alpha} K_{1}|k| \sqrt{2\left|Q_{\infty}\right|}}{\Omega} \mathrm{e}^{-\Omega y_{7}}-\frac{k K_{1}}{\alpha} \mathrm{e}^{-|k| \sqrt{2\left|Q_{\infty}\right|} y_{7}}$,

where $\Omega$ is

$\Omega=\left\{\begin{array}{lc}\mathrm{i} \sqrt{2 k^{2}\left|Q_{\infty}\right|+2|k| \alpha} \quad k<0, \\ \sqrt{2 k \alpha-2 k^{2}\left|Q_{\infty}\right|} \quad k \in\left(0,-\frac{\alpha}{Q_{\infty}}\right), \\ \mathrm{i} \sqrt{2 k^{2}\left|Q_{\infty}\right|-2 k \alpha} \quad k>-\frac{\alpha}{Q_{\infty}},\end{array}\right.$

and $K_{1}$ is defined through

$K_{1}=\frac{|k|}{\sqrt{2\left|Q_{\infty}\right|}} \frac{\bar{f}(k) q^{\frac{2}{3}} A i^{\prime}(0)}{q^{\frac{2}{3}} A i^{\prime}(0)-\frac{(\mathrm{i} k)^{\frac{1}{3}}|k|}{\lambda \mu_{w} \sqrt{2\left|Q_{\infty}\right|}} \int_{0}^{\infty} A i\left(q^{\frac{2}{3}} z^{\prime}\right) \mathrm{d} z^{\prime}}$. 
Relating $\bar{P}_{u}$ to $\bar{p}_{u}$ using the condition (5.6), we have that

$\bar{P}_{u}=\frac{\frac{k^{2}}{\lambda} \bar{A}_{u}+\frac{k}{\alpha} K_{1}|k| \sqrt{2\left|Q_{\infty}\right|}}{\Omega}-\frac{k}{\alpha} K_{1}$.

We now return to our linearised lower deck equations. After performing Fourier transforms and eliminating $\bar{v}_{u}$ (the Fourier transform of $v_{u}$ ) in a similar fashion to the analysis performed for the steady problem, we obtain the boundary value problem for the linearised lower deck variables in Fourier space

$\mathrm{i} \rho_{w} \frac{\mathrm{d} \bar{u}_{u}}{\mathrm{~d} y_{5}}\left(\alpha+\lambda y_{5} k\right)=\mu_{w} \frac{\mathrm{d}^{3} \bar{u}_{u}}{\mathrm{~d} y_{5}^{3}}$

$\bar{u}_{u}=0 \quad$ at $\quad y_{5}=0$,

$\bar{u}_{u}=\bar{A}_{u} \quad$ as $\quad y_{5} \rightarrow \infty$,

$\frac{\mathrm{d}^{2} \bar{u}_{u}}{\mathrm{~d} y_{5}^{2}}=\frac{\mathrm{i} k}{\mu_{w}}\left(\frac{\frac{k^{2}}{\lambda} \bar{A}_{u}+\frac{k}{\alpha} K_{1}|k| \sqrt{2\left|Q_{\infty}\right|}}{\Omega}-\frac{k}{\alpha} K_{1}\right)$ at $y_{5}=0$.

We now make the transformations

$\zeta=\lambda(\mathrm{i} k)^{\frac{1}{3}} y_{5}+\zeta_{0}, \quad \zeta_{0}=\frac{\mathrm{i} \alpha}{(\mathrm{i} k)^{\frac{2}{3}}}$.

At this point, we note that we are required to introduce a branch cut of $(i k)^{\frac{1}{3}}$, which we will again place along the positive imaginary semi-axis in the $k$-plane. Our boundary problem for $\bar{u}_{u}$ is, therefore,

$\frac{\mathrm{d}^{3} \bar{u}_{u}}{\mathrm{~d} \zeta^{3}}-\frac{\rho_{w}}{\lambda \mu_{w}} \zeta \frac{\mathrm{d} \bar{u}_{u}}{\mathrm{~d} \zeta}=0$

$\frac{\mathrm{d}^{2} \bar{u}_{u}}{\mathrm{~d} \zeta^{2}}=\frac{(\mathrm{i} k)^{\frac{1}{3}}}{\mu_{w} \lambda}\left(\frac{\frac{k^{2}}{\lambda} \bar{A}_{u}+\frac{k}{\alpha} K_{1}|k| \sqrt{2\left|Q_{\infty}\right|}}{\Omega}-\frac{k}{\alpha} K_{1}\right)$ at $\zeta=\zeta_{0}$,

$\bar{u}_{u}=\bar{A}_{u}$ as $\zeta \rightarrow \infty$,

$\bar{u}_{u}=0$ at $\zeta=\zeta_{0}$.

The general form of the solution to the ODE above is

$\frac{\mathrm{d} \bar{u}_{u}}{\mathrm{~d} \zeta}=C A i\left(q_{1}^{\frac{2}{3}} \zeta\right)+D B i\left(q_{1}^{\frac{2}{3}} \zeta\right)$

where $q_{1}=\sqrt{\frac{\rho_{w}}{\lambda \mu_{w}}}$, and $A i$ and $B i$ are the Airy functions. In order to satisfy (5.22c), we require $D=0$; otherwise, the derivative of $\bar{u}_{u}$ will be infinite while $\bar{u}_{u}$ is tending to a finite value which is a contradiction. Differentiating this solution once and matching it with $(5.22 \mathrm{~b})$, we have

$q_{1}^{\frac{2}{3}} C A i^{\prime}\left(q_{1}^{\frac{2}{3}} \zeta_{0}\right)=\frac{(\mathrm{i} k)^{\frac{1}{3}}}{\mu_{w} \lambda}\left(\frac{\frac{k^{2}}{\lambda} \bar{A}_{u}+\frac{k}{\alpha} K_{1}|k| \sqrt{2\left|Q_{\infty}\right|}}{\Omega}-\frac{k}{\alpha} K_{1}\right)$. 
In addition, we can integrate (5.23) and considering (5.22d), we obtain

$\bar{u}_{u}=C \int_{\zeta_{0}}^{\zeta} A i\left(q_{1}^{\frac{2}{3}} \zeta^{\prime}\right) \mathrm{d} \zeta^{\prime}$

In the limit $\zeta \rightarrow \infty$, we can deduce from (5.22c) that

$\bar{A}_{u}=C \int_{\zeta_{0}}^{\infty} A i\left(q_{1}^{\frac{2}{3}} \zeta^{\prime}\right) \mathrm{d} \zeta^{\prime}$

Eliminating $C$ using (5.24), we have that $\bar{A}_{u}$ can be expressed in the following form:

$\bar{A}_{u}=\frac{(\mathrm{i} k)^{\frac{1}{3}}}{\alpha} \frac{\lambda k K_{1} R_{1} I_{1}}{\lambda^{2} \mu_{w} q_{1}^{\frac{2}{3}} A i^{\prime}\left(q_{1}^{\frac{2}{3}} \zeta_{0}\right) \Omega-(\mathrm{i} k)^{\frac{1}{3}} k^{2} I_{1}}$,

where we have defined the parameters:

$R_{1}=|k| \sqrt{2\left|Q_{\infty}\right|}-\Omega, \quad I_{1}=\int_{\zeta_{0}}^{\infty} A i\left(q_{1}^{\frac{2}{3}} \zeta^{\prime}\right) \mathrm{d} \zeta^{\prime}$

Substituting this into (5.19), we find

$\bar{P}_{u}=\frac{\lambda^{2} \mu_{w} k K_{1} R_{1} q_{1}^{\frac{2}{3}} A i^{\prime}\left(q_{1}^{\frac{2}{3}} \zeta_{0}\right) \Omega}{\alpha \Omega\left(\lambda^{2} \mu_{w} q_{1}^{\frac{2}{3}} A i^{\prime}\left(q_{1}^{\frac{2}{3}} \zeta_{0}\right) \Omega-(\mathrm{i} k)^{\frac{1}{3}} k^{2} I_{1}\right)}$.

It is now necessary to return to physical space. Performing an inverse Fourier transform for $\bar{P}_{u}$ yields

$\tilde{P}_{5}^{\prime}\left(x_{*}, t\right)=\frac{\mathrm{e}^{\mathrm{i} \alpha t}}{2 \pi} \int_{-\infty}^{\infty} \frac{\lambda^{2} \mu_{w} k K_{1} R_{1} q_{1}^{\frac{2}{3}} A i^{\prime}\left(q_{1}^{\frac{2}{3}} \zeta_{0}\right)}{\alpha\left(\lambda^{2} \mu_{w} q_{1}^{\frac{2}{3}} A i^{\prime}\left(q_{1}^{\frac{2}{3}} \zeta_{0}\right) \Omega-(\mathrm{i} k)^{\frac{1}{3}} k^{2} I_{1}\right)} \mathrm{e}^{\mathrm{i} k x_{*}} \mathrm{~d} k$.

In order to evaluate this integral, we are going to deform the integration contour into the complex plane. In preparation for this, we shall evaluate the poles of the integrand. These occur when we have

$\mu_{w} \lambda^{2} q_{1}^{\frac{2}{3}} A i^{\prime}\left(q_{1}^{\frac{2}{3}} \zeta_{0}\right) \Omega-(\mathrm{i} k)^{\frac{1}{3}} k^{2} \int_{\zeta_{0}}^{\infty} A i\left(q_{1}^{\frac{2}{3}} \zeta^{\prime}\right) \mathrm{d} \zeta^{\prime}=0$

This is a dispersion relation for $k$. In the limit of $k \rightarrow 0$, if we assume that $\zeta_{0}$ remains finite (i.e. $\alpha \sim k^{2 / 3}$ ), then this equation becomes

$A i^{\prime}\left(q_{1}^{\frac{2}{3}} \zeta_{0}\right)=0$

It is known that this equation has an infinite number of roots, all lying on the negative real semi-axis of the $\zeta_{0}$-plane. This suggests that the dispersion relation has an infinite number of roots as well. In order to study the behaviour 


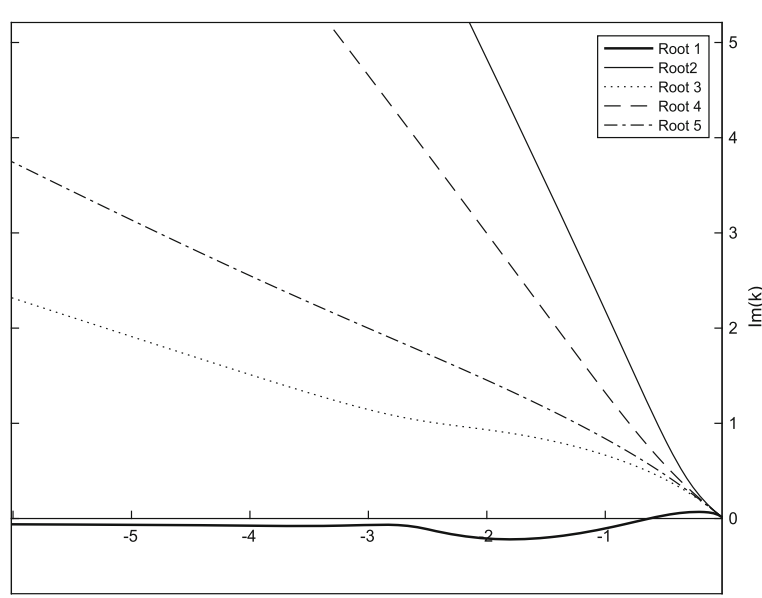

$\operatorname{Re}(\mathrm{k})$

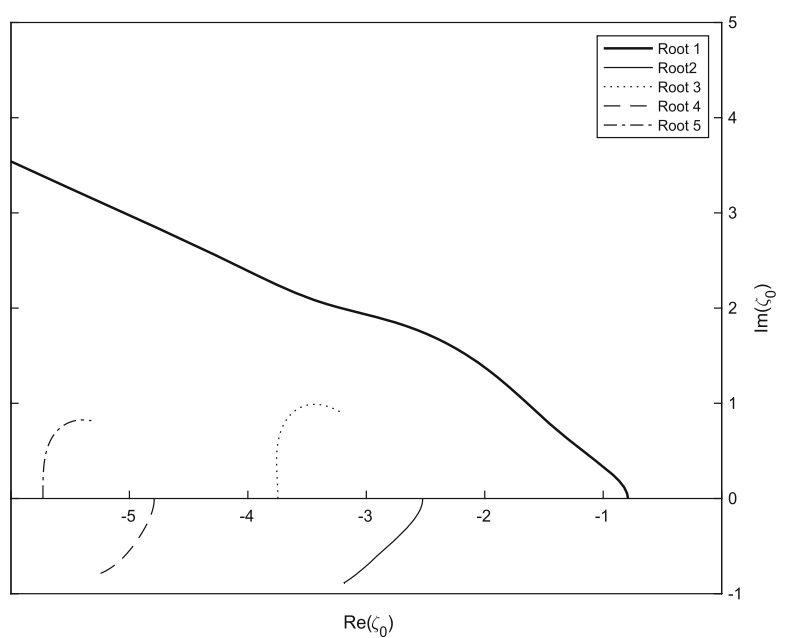

Fig. 2 Behaviour of the first five roots of (5.30) with $Q=-1$ in both the $k$-plane and the $\zeta_{0}$-plane

of the first few roots of this equation, it is only necessary to consider the behaviour of roots in the limit $k \rightarrow \infty$. Assuming that $\zeta_{0}$ again remains finite, this is equivalent to solving

$$
\int_{\zeta_{0}}^{\infty} A i\left(q_{1}^{\frac{2}{3}} \zeta^{\prime}\right) \mathrm{d} \zeta^{\prime}=0
$$

Similar to Eq. (5.31), this equation has infinite roots. It is known from properties of the Airy function that these come in complex conjugate pairs and all lie in the negative real half of the $\zeta_{0}$-plane. It can be seen that the first root of (5.31) behaves differently in the $\zeta_{0}$-plane. As $k \rightarrow \infty$, instead of tending to a finite point which is a root of (5.32), it instead tends to infinity. This root also behaves differently in the $k$-plane. As $\alpha$ goes from zero to infinity, all other roots remain in the second quadrant of the $k$-plane for all $\alpha$. The first root, however, starts in the second quadrant and crosses the negative real semi-axis at a critical point $k_{*}<0$ which has a corresponding critical frequency $\alpha_{*}$. For all $\alpha>\alpha_{*}$, this root remains in the third quadrant. This is the root which corresponds to the wavenumber of the Tollmien-Schlichting wave. Trajectories of the first five roots in both the $k$ and $\zeta_{0}$ planes can be found in Fig. 2, where the root corresponding to the Tollmien-Schlichting wave is in bold.

The process used to calculate the behaviour of the roots as $\alpha$ increased was as follows. First, Eq. (5.30) is written in terms of $\zeta_{0}$ only, using (5.21). Then, Eq. (5.31) is used to find the position of each root at $\alpha=0$. For each subsequent value of $\alpha$, the previous value of $\zeta_{0}$ was used as an initial guess, and the dispersion relation was solved using Newton Iterations.

Returning to (5.29), we deform our contour of integration in the $k$-plane as shown in Fig. 3. We have had to split our contours into the regions of $k<0$ and $k>0$ due to the branch cut we introduced along the positive imaginary semi-axis. We, therefore, split our integral up in the following way:

$\tilde{P}_{5}^{\prime}\left(x_{*}, t\right)=\frac{\mathrm{e}^{\mathrm{i} \alpha t}}{2 \pi}\left(\int_{-\infty}^{0}+\int_{0}^{-\frac{\alpha}{2 Q \infty}}+\int_{-\frac{\alpha}{2 Q \infty}}^{\infty}\right) \frac{\lambda^{2} \mu_{w} k K_{1} R_{1} q_{1}^{\frac{2}{3}} A i^{\prime}\left(q_{1}^{\frac{2}{3}} \zeta_{0}\right)}{\alpha\left(\lambda^{2} \mu_{w} q_{1}^{\frac{2}{3}} A i^{\prime}\left(q_{1}^{\frac{2}{3}} \zeta_{0}\right) \Omega-(\mathrm{i} k)^{\frac{1}{3}} k^{2} I_{1}\right)} \mathrm{e}^{\mathrm{i} k x_{*}} \mathrm{~d} k$. 


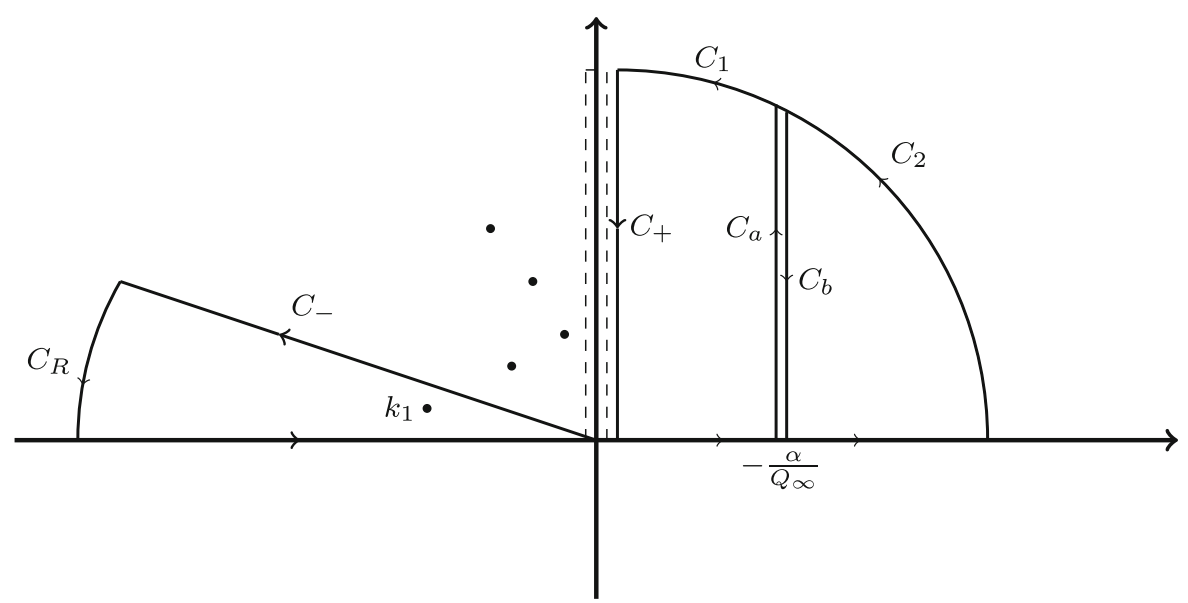

Fig. 3 Deformed integration contour for subsonic flow

If we let the radii of the segments shown in Fig. 3 go to infinity then by Jordan's lemma, we can disregard the integrals along the arcs. Using Watson's lemma, we find that

$$
\begin{aligned}
& \int_{C_{-}} \frac{\lambda^{2} \mu_{w} k K_{1} R_{1} q_{1}^{\frac{2}{3}} A i^{\prime}\left(q_{1}^{\frac{2}{3}} \zeta_{0}\right)}{\left.\alpha \lambda^{2} \mu_{w} q_{1}^{\frac{2}{3}} A i^{\prime}\left(q_{1}^{\frac{2}{3}} \zeta_{0}\right) \Omega-(\mathrm{i} k)^{\frac{1}{3}} k^{2} I_{1}\right)} \mathrm{e}^{\mathrm{i} k x_{*}} \mathrm{~d} k=-\frac{2 \mathrm{i} \bar{f}(0)}{\alpha \sqrt{2\left|Q_{\infty}\right|}} \frac{1}{x_{*}^{3}}, \\
& \int_{C_{+}} \frac{\lambda^{2} \mu_{w} k K_{1} R_{1} q_{1}^{\frac{2}{3}} A i^{\prime}\left(q_{1}^{\frac{2}{3}} \zeta_{0}\right) \mathrm{e}^{\mathrm{i} k x_{*}}}{\alpha\left(\lambda^{2} \mu_{w} q_{1}^{\frac{2}{3}} A i^{\prime}\left(q_{1}^{\frac{2}{3}} \zeta_{0}\right) \Omega-(\mathrm{i} k)^{\frac{1}{3}} k^{2} I_{1}\right)} \mathrm{d} k=\frac{2 \mathrm{i} \bar{f}(0)}{\alpha \sqrt{2\left|Q_{\infty}\right|}} \frac{1}{x_{*}^{3}} .
\end{aligned}
$$

As the integrals along $C_{a}$ and $C_{b}$ cancel out, by the residue theorem, we find that at large values $x$, the TollmienSchlichting wave emerges from the perturbation field with

$$
P_{T S}\left(t, x_{*}\right)=\Lambda\left(\alpha, Q_{\infty}\right) \bar{f}\left(k_{1}\right) \mathrm{e}^{\mathrm{i}\left(\alpha t+k_{1} x\right)}+\cdots
$$

where $\Lambda$ is the receptivity coefficient found to be

$$
\Lambda\left(\alpha, Q_{\infty}\right)=\frac{-\frac{3}{\alpha} \mathrm{i} \lambda^{2} \mu_{w} k_{1} K_{\Lambda} R_{\Lambda} q_{1}^{\frac{2}{3}} A i^{\prime}\left(q_{1}^{\frac{2}{3}} \zeta_{\Lambda}\right)}{2 A i\left(q_{1}^{\frac{2}{3}} \zeta_{\Lambda}\right) \frac{\zeta_{\Lambda}}{k_{1}} C_{\Lambda}+7\left(\mathrm{i} k_{1}\right)^{\frac{1}{3}} k_{1} I_{\Lambda}-\frac{3\left(2\left|Q_{\infty}\right| k_{1}+\alpha\right)}{\Omega_{\Lambda}} \lambda^{2} \mu_{w} q_{1}^{\frac{2}{3}} A i^{\prime}\left(q_{1}^{\frac{2}{3}} \zeta_{\Lambda}\right)}
$$

with the parameters with subscript $\Lambda$ defined as follows:

$$
\begin{aligned}
& \Omega_{\Lambda}=\sqrt{-2 k_{1}^{2}\left|Q_{\infty}\right|-2\left|k_{1}\right| \alpha}, \quad R_{\Lambda}=\left|k_{1}\right| \sqrt{2\left|Q_{\infty}\right|}-\Omega_{\Lambda}, \\
& K_{\Lambda}=\frac{\left|k_{1}\right|}{\sqrt{2\left|Q_{\infty}\right|}} \frac{q^{\frac{2}{3}} A i^{\prime}(0)}{q^{\frac{2}{3}} A i^{\prime}(0)-\frac{\left(\mathrm{i} k_{1}\right)^{\frac{1}{3}}\left|k_{1}\right|}{\lambda \mu_{w} \sqrt{2\left|Q_{\infty}\right|}} \int_{0}^{\infty} A i\left(q^{\frac{2}{3}} z^{\prime}\right) \mathrm{d} z^{\prime}},
\end{aligned}
$$



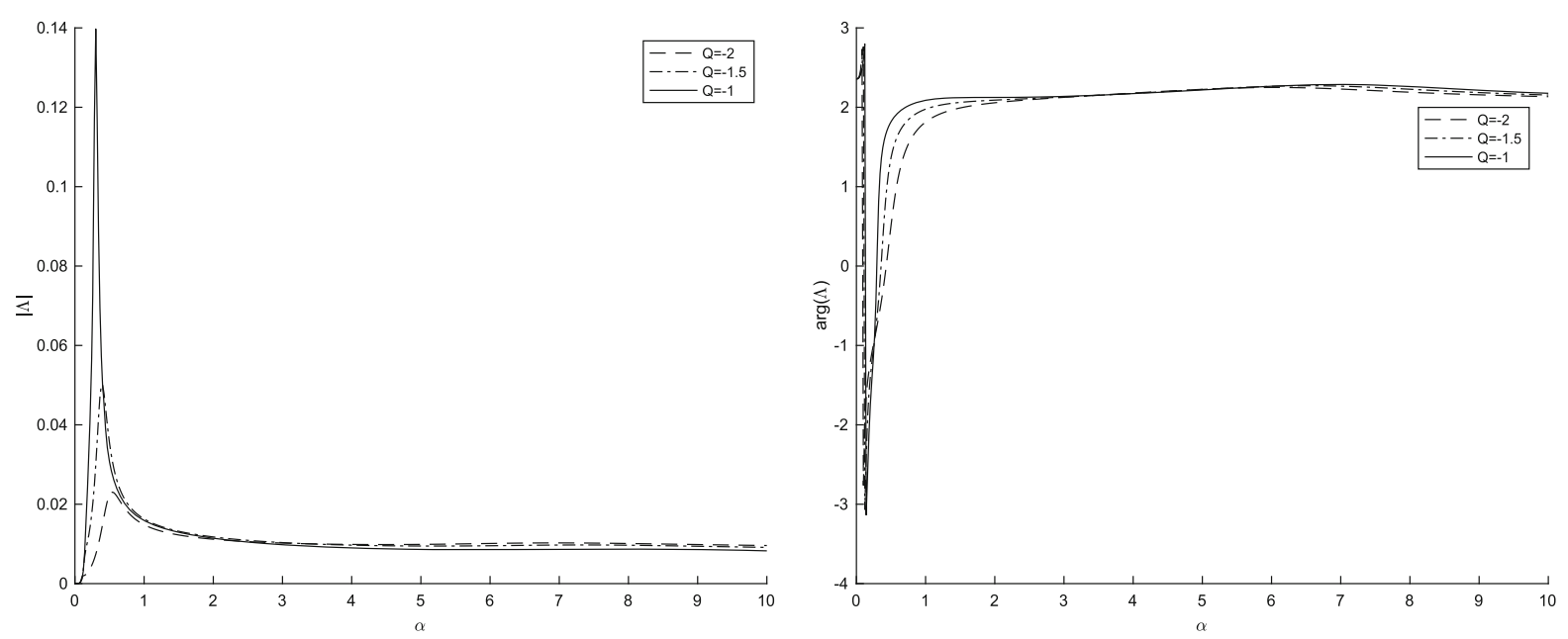

Fig. 4 Subsonic receptivity coefficient modulus and argument

$$
\zeta_{\Lambda}=\frac{(\mathrm{i} \alpha)}{\left(\mathrm{i} k_{1}\right)^{\frac{2}{3}}}, \quad I_{\Lambda}=\int_{\zeta_{\Lambda}}^{\infty} A i\left(q_{1}^{\frac{2}{3}} \zeta^{\prime}\right) \mathrm{d} \zeta^{\prime}, \quad C_{\Lambda}\left(2\left(\mathrm{i} k_{1}\right)^{\frac{1}{3}} k_{1}^{2}-\lambda^{2} \mu_{w} \Omega_{\Lambda} q^{\frac{7}{3}} \zeta_{\Lambda}\right) .
$$

Here, it is important to note that the receptivity coefficient is a function of the Kármán-Guderley parameter $Q_{\infty}$ and frequency $\alpha$ only, and does not depend on the roughness shape. Numerical calculations of the receptivity coefficient can be found in Fig. 4.

\subsubsection{Supersonic flow}

For supersonic flow, the analysis proceeds in much the same way. The solution to the problem (5.15) in this case is

$\bar{p}_{u}=\frac{\frac{k^{2}}{\lambda} \bar{A}_{u}+\mathrm{i} \frac{k^{2}}{\alpha} K_{2} \sqrt{2\left|Q_{\infty}\right|}}{\Omega} \mathrm{e}^{-\Omega y_{7}}-\frac{k}{\alpha} K_{2} \mathrm{e}^{-\mathrm{i} k \sqrt{2 Q_{\infty}} y_{7}}$,

where $\Omega$ is now defined to be

$\Omega=\left\{\begin{array}{lc}\sqrt{2 k^{2} Q_{\infty}-2|k| \alpha} & k<-\frac{\alpha}{Q_{\infty},} \\ \mathrm{i} \sqrt{2|k| \alpha-2 k^{2} Q_{\infty}} & k \in\left(-\frac{\alpha}{Q_{\infty}}, 0\right), \\ \sqrt{2 k \alpha+2 k^{2} Q_{\infty}} & k>0,\end{array}\right.$

and $K_{2}$ is

$$
K_{2}=\frac{\mathrm{i} k}{\sqrt{2 Q_{\infty}}} \frac{\bar{f}(k) q^{\frac{2}{3}} A i^{\prime}(0)}{q^{\frac{2}{3}} A i^{\prime}(0)+i \frac{(\mathrm{i} k)^{\frac{1}{3}} k}{\lambda \mu_{w} \sqrt{2 Q_{\infty}}} \int_{0}^{\infty} A i\left(q^{\frac{2}{3}} z^{\prime}\right) \mathrm{d} z^{\prime}} .
$$

Performing an identical analysis to the subsonic case, we find that for $Q_{\infty}>0$

$$
\bar{A}_{u}=\frac{\lambda(\mathrm{i} k)^{\frac{1}{3}} k K_{2} R_{2} I_{1}}{\alpha\left(\lambda^{2} \mu_{w} q_{1}^{\frac{2}{3}} A i^{\prime}\left(q_{1}^{\frac{2}{3}} \zeta_{0}\right) \Omega-(\mathrm{i} k)^{\frac{1}{3}} k^{2} I_{1}\right)},
$$




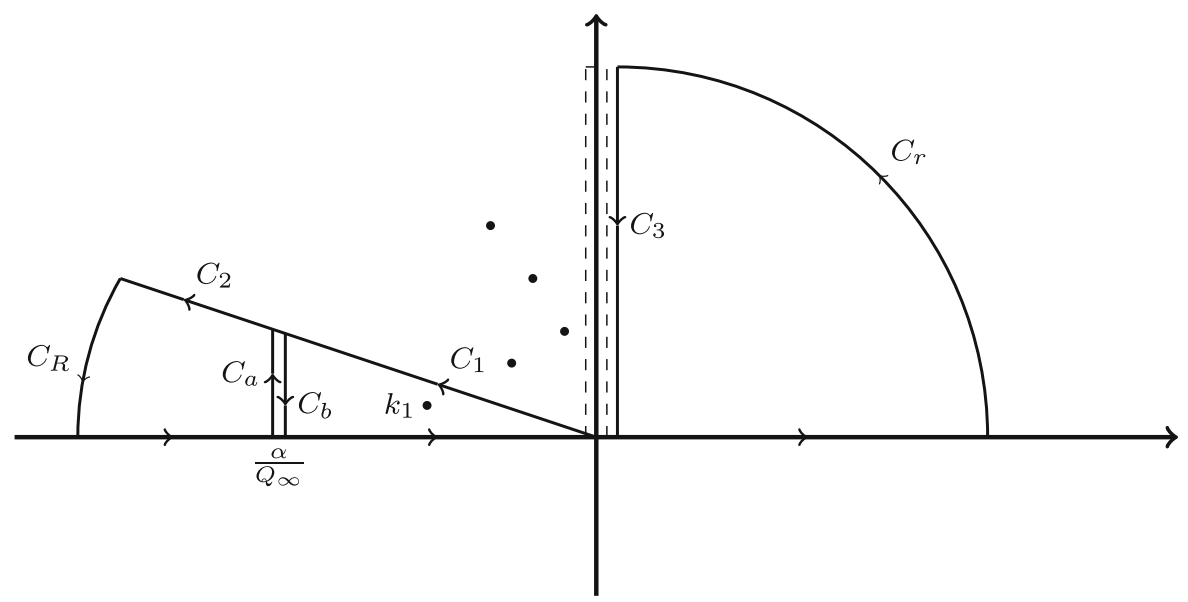

Fig. 5 Deformed integration contour in the $k$-plane for supersonic flow

$\bar{P}_{u}=\frac{q_{1}^{\frac{2}{3}} \lambda^{2} \mu_{w} A i^{\prime}\left(q_{1}^{\frac{2}{3}} \zeta_{0}\right) k K_{2} R_{2}}{\alpha\left(\lambda^{2} \mu_{w} q_{1}^{\frac{2}{3}} A i^{\prime}\left(q_{1}^{\frac{2}{3}} \zeta_{0}\right) \Omega-(\mathrm{i} k)^{\frac{1}{3}} k^{2} I_{1}\right)}$,

where $q_{1}, \zeta_{0}$, and $I_{1}$ are the same as in the subsonic case and $R_{2}$ is defined through

$R_{2}=\mathrm{i} k \sqrt{2 Q_{\infty}}-\Omega$.

Performing an inverse Fourier transform in order to return back to physical space, we have

$\tilde{P}_{5}^{\prime}\left(t, x_{*}\right)=\frac{\mathrm{e}^{\mathrm{i} \alpha t}}{2 \pi} \int_{-\infty}^{\infty} \frac{q_{1}^{\frac{2}{3}} \lambda^{2} \mu_{w} A i^{\prime}\left(q_{1}^{\frac{2}{3}} \zeta_{0}\right) k K_{2} R_{2}}{\alpha\left(\lambda^{2} \mu_{w} q_{1}^{\frac{2}{3}} A i^{\prime}\left(q_{1}^{\frac{2}{3}} \zeta_{0}\right) \Omega-(\mathrm{i} k)^{\frac{1}{3}} k^{2} I_{1}\right)} \mathrm{e}^{\mathrm{i} k x_{*}} \mathrm{~d} k$.

This yields the dispersion relation for supersonic flow

$\lambda^{2} \mu_{w} q_{1}^{\frac{2}{3}} A i^{\prime}\left(q_{1}^{\frac{2}{3}} \zeta_{0}\right) \Omega-(i k)^{\frac{1}{3}} k^{2} \int_{\zeta_{0}}^{\infty} A i\left(q_{1}^{\frac{2}{3}} \zeta^{\prime}\right) \mathrm{d} \zeta^{\prime}=0$

This is nearly identical to the dispersion relation for subsonic flow (see 5.30), except for change in definition of $\Omega$. The roots of this equation behave in a similar manner in both the $k$ and $\zeta_{0}$ planes as in the subsonic case. In order to analyse the integral (5.46), we again split the integral into three regions and deform each line into a contour in the complex $k$-plane. The deformed integration contour can be seen in Fig. 5. The behaviour of poles of the integrand is the same as in the subsonic case. We note that it does not matter which contour contains the root $k_{1}$. Again, we can disregard the integral along both arcs in view of Jordan's Lemma, and making use of Watson's Lemma, we find the integrals along $C_{1}, C_{2}$, and $C_{3}$ sum to zero at large values of $x_{*}$. Therefore, using residue calculus, we have that for supersonic flow, the Tollmien-Schlichting wave emerges from the perturbation field such that

$P_{T S}=\Lambda\left(\alpha, Q_{\infty}\right) \bar{f}\left(k_{1}\right) \mathrm{e}^{\mathrm{i}\left(\alpha t+k_{1} x_{*}\right)}+\cdots$, 

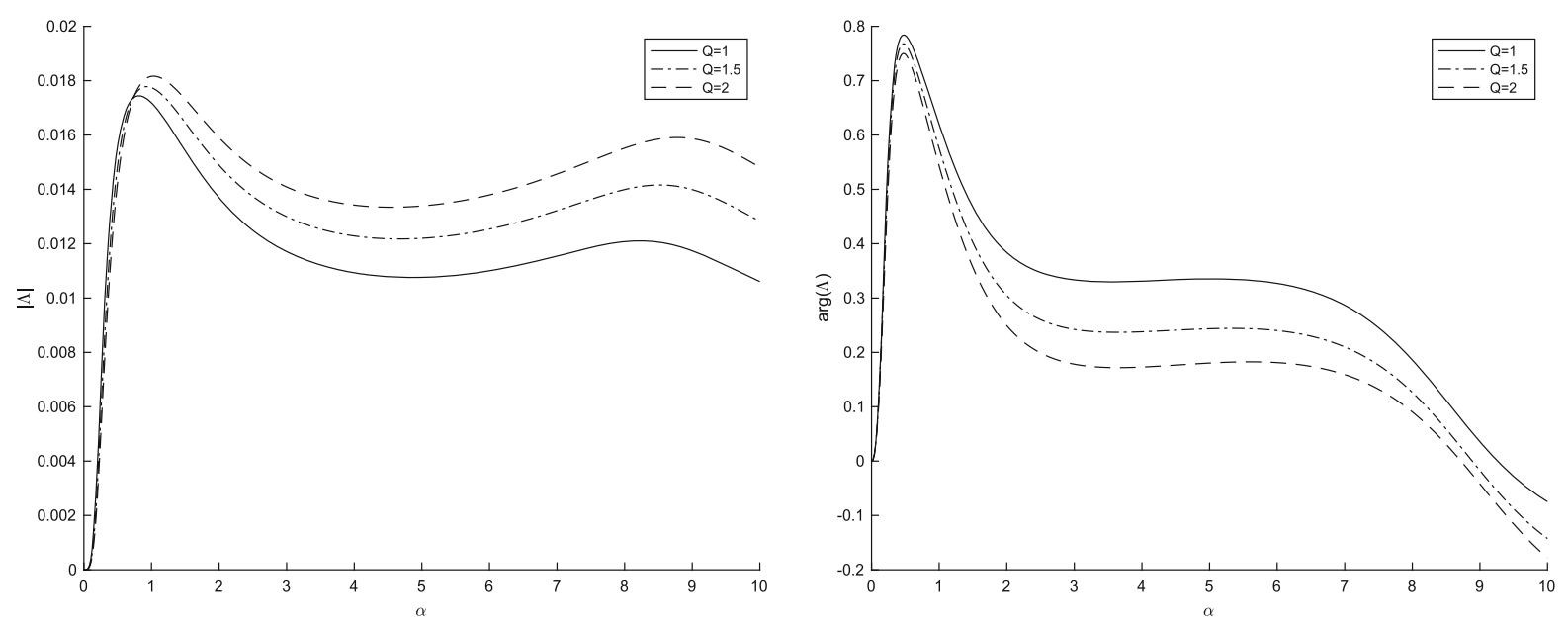

Fig. 6 Supersonic receptivity coefficient modulus and argument

where in this case, the receptivity coefficient $\Lambda$ is

$\Lambda\left(\alpha, Q_{\infty}\right)=\frac{\frac{3}{\alpha} \mathrm{i} \lambda^{2} \mu_{w} k_{1} K_{\Lambda} R_{\Lambda} q_{1}^{\frac{2}{3}} A i^{\prime}\left(q_{1}^{\frac{2}{3}} \zeta_{\Lambda}\right)}{2 A i\left(q_{1}^{\frac{2}{3}} \zeta_{\Lambda}\right) \frac{\zeta_{\Lambda}}{k_{1}} C_{\Lambda}+7\left(\mathrm{i} k_{1}\right)^{\frac{1}{3}} k_{1} I_{\Lambda}-\frac{3\left(2 Q_{\infty} k_{1}+\alpha\right)}{\Omega_{\Lambda}} \lambda^{2} \mu_{w} q_{1}^{\frac{2}{3}} A i^{\prime}\left(q_{1}^{\frac{2}{3}} \zeta_{\Lambda}\right)}$

with the coefficients having the subscript $\Lambda$ defined through

$\Omega_{\Lambda}=\mathrm{i} \sqrt{2\left|k_{1}\right| \alpha-2 k_{1}^{2} Q_{\infty}}, \quad R_{\Lambda}=\mathrm{i} k_{1} \sqrt{2 Q_{\infty}}-\Omega_{\Lambda}$,
$K_{\Lambda}=\frac{\mathrm{i} k_{1}}{\sqrt{2 Q_{\infty}}} \frac{q^{\frac{2}{3}} A i^{\prime}(0)}{q^{\frac{2}{3}} A i^{\prime}(0)-i \frac{\left(\mathrm{i} k_{1}\right)^{\frac{1}{3}} k_{1}}{\lambda \mu_{w} \sqrt{2 Q_{\infty}}} \int_{0}^{\infty} A i\left(q^{\frac{2}{3}} z^{\prime}\right) \mathrm{d} z^{\prime}}$,
$\zeta_{\Lambda}=\frac{(\mathrm{i} \alpha)}{\left(i k_{1}\right)^{\frac{2}{3}}}, \quad I_{\Lambda}=\int_{\zeta_{\Lambda}}^{\infty} A i\left(q_{1}^{\frac{2}{3}} \zeta^{\prime}\right) \mathrm{d} \zeta^{\prime}, \quad C_{\Lambda}=\left(2\left(\mathrm{i} k_{1}\right)^{\frac{1}{3}} k_{1}^{2}-\lambda^{2} \mu_{w} \Omega_{\Lambda} q^{\frac{7}{3}} \zeta_{\Lambda}\right)$.

While similar to the subsonic case, we note that the receptivity coefficient for supersonic flow is not identical to (5.36). The results of the numerical calculations for receptivity coefficient in supersonic flow can be found in Fig. 6.

\section{Summary}

This paper studied the receptivity of the Blasius boundary layer to free-stream turbulence in transonic flow. As the receptivity analysis is local, it is, therefore, applicable to an arbitrary boundary layer, for example, a wing surface. We have seen that, unlike acoustic waves, vorticity waves do not produce pressure perturbations to leading order in the free stream or the vorticity deformation layer. Therefore, the vorticity waves cannot penetrate into the boundary layer, so the process by which Tollmien-Schlichting waves are generated differs greatly from the acoustic case. In the present case, Tollmien-Schlichting waves arise due to the interaction between the free-stream turbulence and a roughness element on the plate surface. In order for this interaction to take place, we required that the strict double resonance condition associated with Tollmien-Schlichting waves be followed, i.e. the frequency of the vorticity wave is close to the frequency of the Tollmien-Schlichting wave, and in the Fourier spectrum of the perturbations 
produced by the roughness element is a wave number matching the wave number of the Tollmien-Schlichting wave. Unlike in the case of the acoustic wave, the interaction of the vorticity wave with steady perturbations (produced by the roughness element) takes place in the upper tier of the triple-deck structure.

After first non-dimensionalising the governing equations, solutions in the flow upstream of the roughness element were derived. Due to the lack of leading order pressure perturbations, it was necessary to introduce a transition layer at the outer edge of the boundary layer in which the velocity perturbations caused by the free-stream turbulence decayed. The analysis of the boundary layer was, therefore, simple, as there was no need to introduce a Stokes layer close to the plate as is necessary in the case of acoustic waves.

To analyse the flow around the roughness, we used the scalings corresponding to transonic viscous-invisicid interaction theory, which enabled equations governing the velocity and pressure distributions in the three regions of the triple-deck structure to be derived. We note at this point that while the analysis performed on the lower and middle decks is similar to previous research, the inclusion of free-stream turbulence meant that the asymptotic expansions of the upper deck were novel. The external perturbations present in this deck interacted with the perturbations caused by the roughness element to produce the Tollmien-Schlichting wave.

When finding the amplitude of the Tollmien-Schlichting wave, it was necessary to assume that the height of the roughness element was small compared to $L \mathrm{Re}^{-\frac{11}{18}}$ in order to make analytic progress. This assumption allowed the equations for the lower deck of the triple-deck model to be linearised, so the resulting viscous-inviscid interaction problems could be solved analytically. The behaviour of Tollmien-Schlichting waves far downstream of the roughness element is of primary interest when modelling laminar-turbulent transition as noted in the Introduction. By first solving the steady problem, and then assuming the unsteady solutions were periodic in time before making use of Fourier transforms, elementary results from complex analysis, and Watson's Lemma, a formula for the amplitude of Tollmien-Schlichting waves in this limit was found, depending only on the frequency of the vorticity wave and the Kármán-Guderley parameter. It is interesting that unlike the acoustic case studied by [7], the receptivity coefficient did depend on the sign of the Kármán-Guderley parameter.

To conclude, in this paper, we have derived an explicit equation for the amplitude of Tollmien-Schlichting waves generated by free-stream turbulence in transonic flow far downstream of roughness. This first required solving the Navier-Stokes equations in the various regions upstream of the roughness, before introducing the triple-deck model of $[8,9]$, the use of which allowed the equations governing the flow around the roughness element to be derived. The receptivity coefficient for both subsonic and supersonic flow were found and calculated numerically, and depended only on the frequency of the vorticity wave and the Kármán-Guderley parameter.

Of course, the generation of the Tollmien-Schlichting waves can be also studied by means of the direct numerical simulations of the Navier-Stokes equations. However, a clear advantage of the asymptotic theory presented here is that it provides an explicit dependence of the amplitude of the Tollmien-Schlichting wave on the shape of the wall roughness. We see that in order to delay the laminar-turbulent transition, one needs to minimise the Fourier transform of the wall roughness function at a particular value of the wave number that corresponds to the TollmienSchlichting wave. This result may be directly used to improve the wing design. For example, of particular interest is a small gap between the nasal retractable section of the wing with the main body of the wing. While it cannot be avoided without increasing the landing speed of an aircraft, the effect of this gap can be minimised by simply changing the gap width.

Open Access This article is licensed under a Creative Commons Attribution 4.0 International License, which permits use, sharing, adaptation, distribution and reproduction in any medium or format, as long as you give appropriate credit to the original author(s) and the source, provide a link to the Creative Commons licence, and indicate if changes were made. The images or other third party material in this article are included in the article's Creative Commons licence, unless indicated otherwise in a credit line to the material. If material is not included in the article's Creative Commons licence and your intended use is not permitted by statutory regulation or exceeds the permitted use, you will need to obtain permission directly from the copyright holder. To view a copy of this licence, visit http://creativecommons.org/licenses/by/4.0/. 


\section{References}

1. Schubauer GB, Skramstad HK (1948) Laminar boundary oscillations and transition on a flat plate. NACA Rep 38:251-292

2. Fedorov AV (1982) Generation of instability waves in a boundary-layer flow of compressbile gas exposed to an acoustic field. Num Meth Continuum Mech (Novosibirsk) 13:106

3. Goldstein ME (1983) The evolution of Tollmien-Schlichting waves near a leading edge. J Fluid Mech 127:59-81

4. Goldstein ME (1985) Scattering of acoustic waves into Tollmien-Schlichting waves by small streamwise variations in surface geometry. J Fluid Mech 154:509-529

5. Ruban AI (1984) On the generation of Tollmien-Schlichting waves by sound. Fluid Dyn 19:709-717

6. Kachanov YS, Kozlov VV, Levchenko VY (1982) Beginning of turbulence in the boundary layer. Nauka, Novosibirsk

7. Ruban AI, Bernots T, Kravtsova MA (2016) Linear and nonlinear receptivity of the boundary layer in transonic flows. J Fluid Mech 786:154-189

8. Bowles RI, Smith FT (1993) On boundary-layer transition in transonic flow. J Eng Math 27:309-342

9. Timoshin S (1990) Asymptotic form of the lower branch of the neutral curve in transonic flow. Uch Zap TsAGI 21(6):50-57

10. Hunt JCR, Graham JMR (1978) Free-stream vorticity near plate boundaries. J Fluid Mech 84:209-235

11. Guliaev AN, Kozlov VE, Kuznetzov VR, Mineev BI, Secundov AN (1989) Interaction of a laminar boundary layer with external turbulence. Fluid Dyn 24:700-710

12. Duck PW, Ruban AI, Zhikharev CN (1996) The generation of Tollmien-Schlichting waves by free-stream turbulence. J Fluid Mech 312:341-371

Publisher's Note Springer Nature remains neutral with regard to jurisdictional claims in published maps and institutional affiliations. 